\title{
Synthesis of a mannose nonasaccharide existing in the exopolysaccharide of Cryphonectria parasitica
}

\author{
Jianjun Zhang, Zuchao Ma, Fanzuo Kong* \\ Research Center for Eco-Environmental Sciences, Academia Sinica, P.O. Box 2871, Beijing 100085, PR China
}

Received 31 March 2003; accepted 4 June 2003

\begin{abstract}
$\alpha$-D-Manp - $(1 \rightarrow 2)-\alpha-D-M a n p-(1 \rightarrow 2)-\alpha-D-M a n p-(1 \rightarrow 6)[\alpha-D-M a n p-(1 \rightarrow 3)-\alpha-D-M a n p-(1 \rightarrow 2)-\alpha-D-M a n p-(1 \rightarrow 2)]-\alpha-D-M a n p-$

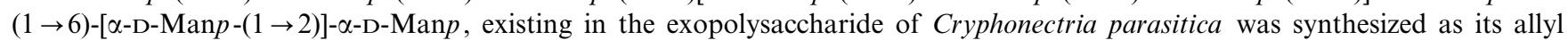
glycoside in a regio- and stereoselective manner.

(C) 2003 Elsevier Ltd. All rights reserved.
\end{abstract}

Keywords: Oligosaccharide; Mannose; Synthesis

\section{Introduction}

Exopolysaccharides (EPSs) of fungi are thought to be agents of phototoxicity and can play a role in plantfungal interactions. ${ }^{1}$ Cryphonectria parasitica (Murr.) Barr is the causal agent of chestnut blight, ${ }^{2}$ which is characterized by the formation of a 'gelatinous zone' beyond the advancing edge of the mycelium. The structure of a new EPS from the virulent strain of $C$. parasitica was elucidated ${ }^{3}$ by means of $2 \mathrm{D}$ NMR spectroscopy and mild hydrolysis and acetolysis. The polysaccharide has a rather complex structure that can be idealized as follows:

As an ongoing project for investigation of the structure-function relationships of oligosaccharides, we have reported the synthesis of a variety of biologically important oligosaccharides, such as $(1 \rightarrow 3)$ branched $(1 \rightarrow 6)$-linked glucans, ${ }^{4} \quad(1 \rightarrow 6)$-branched $(1 \rightarrow 3)$-linked glucans, ${ }^{5}(1 \rightarrow 2)$-branched $(1 \rightarrow 6)$-linked mannans, ${ }^{6} 3,6$-branched mannans ${ }^{7}$ etc. Now we present the synthesis of the mannose nonasaccharide existing in the idealized structure of the EPSs of $C$. parasitica.

\footnotetext{
* Corresponding author. Tel.: +86-10-62936613; fax: +8610-62923563.

E-mail address: fzkong@mail.rcees.ac.cn (F. Kong).
}

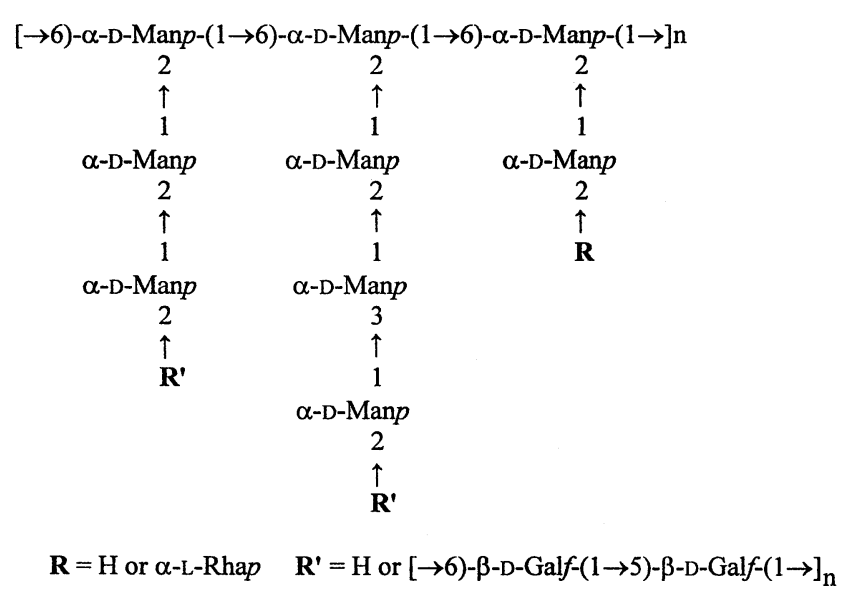

\section{Results and discussion}

As outlined in Scheme 1, selective acetylation of allyl 4,6- $O$-benzylidene- $\alpha$-D-mannopyranoside (1) with acetyl chloride in pyridine gave allyl 3- $O$-acetyl-4,6- $O$-benzylidene- $\alpha$-D-mannopyranoside (2) in satisfactory yield $(91 \%)$, and no acetyl migration was observed. Meanwhile, selective glycosylation of $\mathbf{1}$ with perbenzoylated mannosyl trichloroacetimidate afforded the $(1 \rightarrow 3)$ linked disaccharide $7(76 \%)$. The regioselectivity was confirmed by benzoylation of 7 to give 8 , and the ${ }^{1} \mathrm{H}$ 

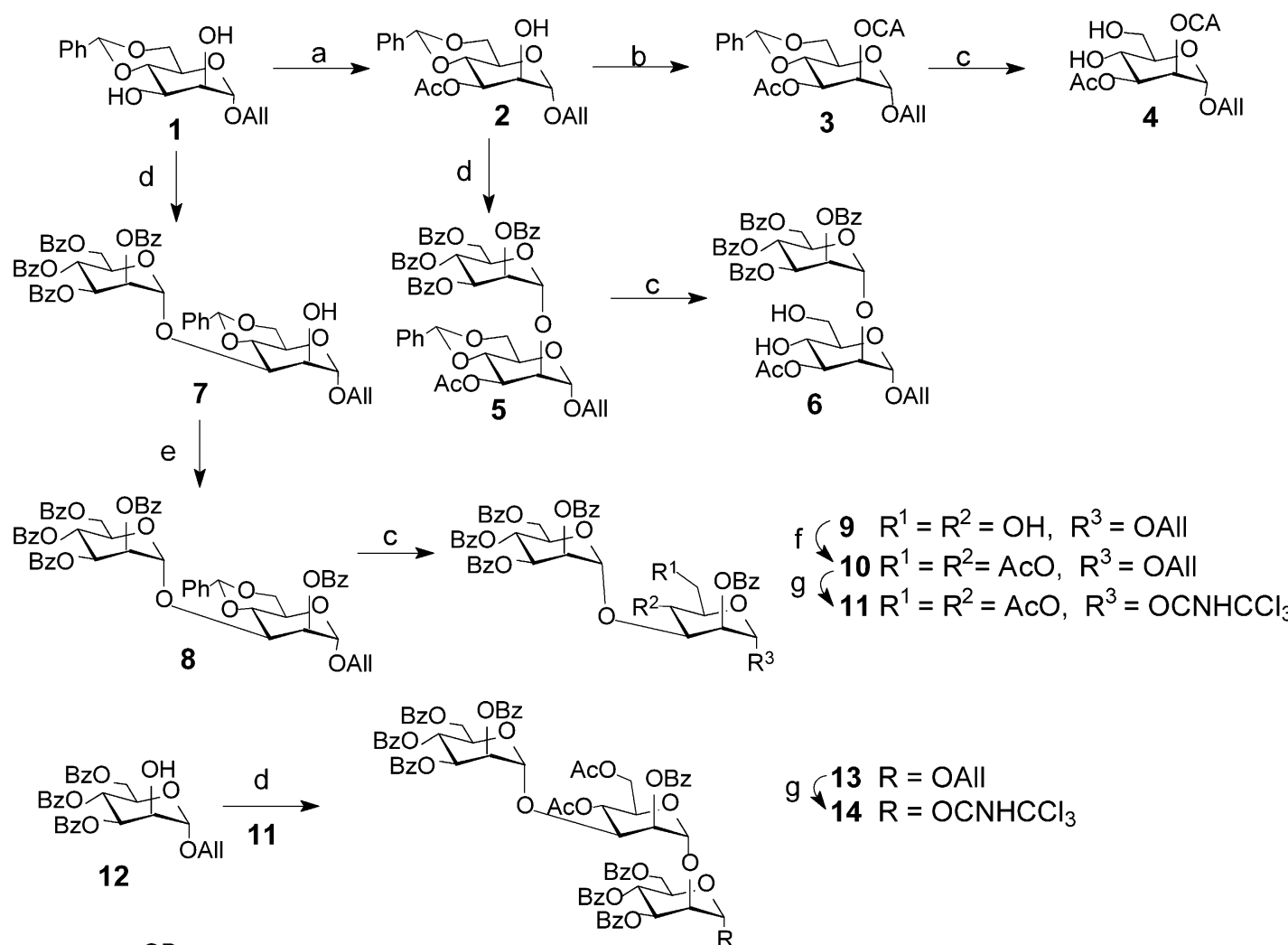

$g\left(\begin{array}{ll}13 & R=O A l l \\ 14 & R=O C N \mathrm{OCCl}_{3}\end{array}\right.$

12
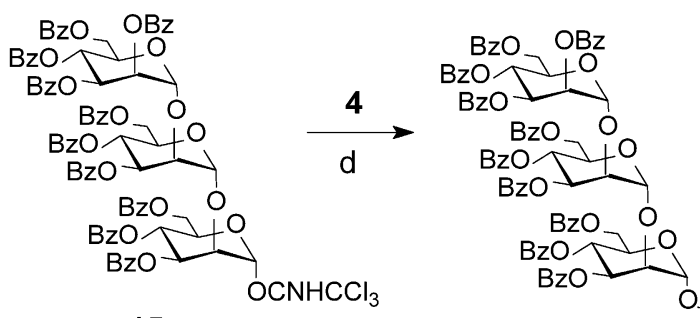

$$
\begin{aligned}
& \text { f } 16 \mathrm{R}^{1}=\mathrm{OH}, \mathrm{R}^{2}=\mathrm{OCA} \\
& 17 R^{1}=\text { OAC, } R^{2}=O C A \\
& h \sqrt{18 R^{1}=O A c,} R^{2}=O H
\end{aligned}
$$

15

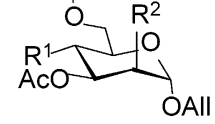

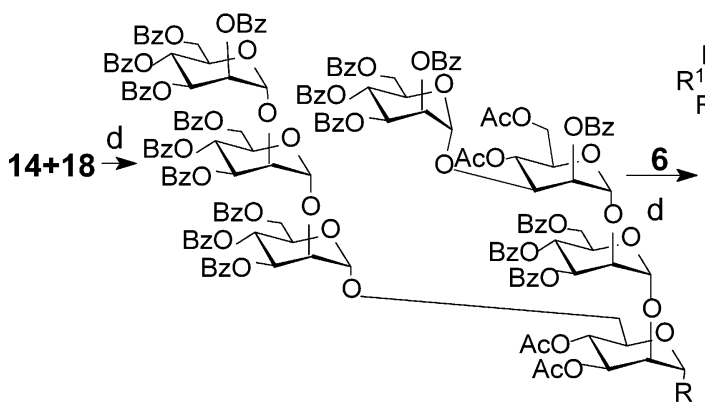

$$
g\left(\begin{array}{ll}
19 & \mathrm{R}=\mathrm{OAll} \\
20 & \mathrm{R}=\mathrm{OCNHCCl}_{3}
\end{array}\right.
$$

$$
\text { i } \begin{cases}21 & R^{1}=B z, R^{2}=A c, R^{3}=H \\ 22 & R^{1}=B z, R^{2}=R^{3}=A c \\ 23 & R^{1}=R^{2}=R^{3}=H\end{cases}
$$

Scheme 1. (a) $\mathrm{CH}_{3} \mathrm{COCl}, \mathrm{CH}_{2} \mathrm{Cl}_{2}$, pyridine; (b) $\mathrm{ClCH}_{2} \mathrm{COCl}, \mathrm{CH}_{2} \mathrm{Cl}_{2}$, pyridine; (c) $90 \% \mathrm{TFA}, 2 \mathrm{~h}, \mathrm{rt}$; (d) TMSOTf, $\mathrm{CH}_{2} \mathrm{Cl}_{2}$; (e) $\mathrm{BzCl}$-pyridine (dry); (f) $\mathrm{Ac}_{2} \mathrm{O}$-pyridine (dry); (g) $\mathrm{PdCl}_{2}, 90 \%$ HOAc-NaOAc, rt, 12 h; then $\mathrm{Cl}_{3} \mathrm{CN}_{2} \mathrm{DBU}_{2} \mathrm{CH}_{2} \mathrm{Cl}_{2} 2-4$ h; (h) thiourea in $\mathrm{EtOH}-\mathrm{CH}_{2} \mathrm{Cl}_{2}$; (i) satd $\mathrm{NH}_{3}-\mathrm{MeOH}, \mathrm{rt}, 72 \mathrm{~h}$.

NMR spectrum of $\mathbf{8}$ showed two characteristic signals for $\mathrm{H}-2$ and $\mathrm{H}-2^{\prime}$ at $\delta 5.63\left(J_{1,2} 1.4, J_{2,3} 3.2 \mathrm{~Hz}\right)$ and 5.76 $\left(J_{1,2} 0.9, J_{2,3} 2.8 \mathrm{~Hz}\right) \mathrm{ppm}$, respectively. Debenzylidenation of $\mathbf{8}$, followed by acetylation, furnished $\mathbf{1 0}(82 \%$ for two steps). Subsequent deallylation and trichloroacetimidation yielded the disaccharide donor 11 ( $83 \%$ for two steps). Condensation of $\mathbf{1 1}$ with allyl 3,4,6-tri- $O$ benzoyl- $\alpha$-D-mannopyranoside (12) gave a trisaccharide 
13. Reiteration of the deallylation and trichloroacetimidation transformed $\mathbf{1 3}$ to the trisaccharide donor 14, which was used to build one side chain. For construction of the other side chain, $\mathbf{2}$ was chloroacetylated to afford 3, and subsequent debenzylidenation furnished the monosaccharide acceptor 4 . Selective 6-O-glycosylation $^{8}$ of $\mathbf{4}$ with perbenzoylated $(1 \rightarrow 2)$-linked trisaccharide donor $\mathbf{1 5}^{\mathbf{7 b}}$ gave $(1 \rightarrow 6)$-linked tetrasaccharide $\mathbf{1 6}$ $(88 \%)$. The selective $6-\mathrm{O}$-glycosylation was verified by acetylation of 16 to furnish 17, and the ${ }^{1} \mathrm{H}$ NMR spectrum of 17 showed a newly emerged signal at $\delta 5.45$ with $J_{3,4}=J_{4,5}=9.9 \mathrm{~Hz}$ for $\mathrm{H}-4$ compared to $\mathbf{1 6}$. Subsequent dechloroacetylation of $\mathbf{1 7}$ with thiourea gave the tetrasaccharide acceptor 18 (84\%). Condensation of 18 with 14 was carried out smoothly giving the heptasaccharide 19 (68\%). Deallylation of 19, followed by trichloroacetimidation, afforded the heptasaccharide donor 20 (78\%). For completion of the assembly of the nonasaccharide, another disaccharide unit was prepared. Thus, coupling of $\mathbf{2}$ with perbenzoylated mannosyl trichloroacetimidate furnished the disaccharide $\mathbf{5}$, and subsequent debenzylidenation afforded the disaccharide acceptor $6(86 \%)$. The nonasaccharide 21 was readily obtained by $6-\mathrm{O}$-selective glycosylation of the disaccharide acceptor $\mathbf{6}$ with the heptasaccharide donor $20(75 \%)$. Acetylation of $\mathbf{2 1}$ gave 22, whose ${ }^{1} \mathrm{H}$ spectrum showed one more signal, compared to 21 , at $\delta 5.32 \mathrm{ppm}$ with $J_{3,4}=J_{4,5}=9.9 \mathrm{~Hz}$ for $\mathrm{H}-4$, confirming the selective 6-O-glycosylation. Deacylation of 22 was carried out in a saturated solution of ammonia in methanol giving the target nonasaccharide 23, and the ${ }^{1} \mathrm{H}$ and ${ }^{13} \mathrm{C}$ NMR spectra of $\mathbf{2 3}$ showed characteristic signals such as at $\delta 5.16,5.16,5.14,5.01,4.99,4.98,4.96,4.91$, and 4.88 for $9 \mathrm{H}-1 \mathrm{~s}$, and $\delta 104.9,104.8,104.8,104.7,103.2,103.2$, $100.7,100.6,100.2$ for $9 \mathrm{C}-1 \mathrm{~s}$, and $\delta 81.39,81.38,81.37$, $81.02,81.01$, and 80.51 for glycosylated $5 \mathrm{C}-2 \mathrm{~s}$ and $1 \mathrm{C}-$ 3 . Since only six signals were found at $\delta 80-82$, and the rest of the C-2 to C-6 signals were all at $<\delta 76$, the 6-Oglycosylation of $\mathbf{6}$ was again confirmed by this; otherwise, if C-4 were glycosylated, a signal at $\delta>80$ would have appeared.

In summary, a complex branched mannose nonasaccharide was synthesized in a regio- and stereoselective way by a simple procedure. Large-scale preparations should be possible with this method.

\section{Experimental}

\subsection{General methods}

Melting points were determined with a 'Mel-Temp' apparatus. Optical rotations were determined with a Perkin-Elmer model 241-MC automatic polarimeter for solutions in a $1-\mathrm{dm}$, jacketed cell. ${ }^{1} \mathrm{H},{ }^{13} \mathrm{C}$, and $2 \mathrm{D}$ NMR spectra were recorded with Varian XL-400 spectro- meters for solutions in $\mathrm{CDCl}_{3}$ or in $\mathrm{D}_{2} \mathrm{O}$ as indicated. Chemical shifts are expressed in ppm downfield from the $\mathrm{Me}_{4} \mathrm{Si}$ absorption. Mass spectra were recorded with a VG PLATFORM mass spectrometer using the electrospray-ionization (ESI) mode. Thin-layer chromatography (TLC) was performed on silica gel HF with detection by charring with $30 \%(\mathrm{v} / \mathrm{v})$ sulfuric acid in $\mathrm{MeOH}$ or by UV detection. Column chromatography was conducted by elution of a column $(8 \times 100,16 \times$ $240,18 \times 300,35 \times 400 \mathrm{~mm})$ of silica gel $(100-200$ mesh) with EtOAc-petroleum ether (bp $60-90{ }^{\circ} \mathrm{C}$ ) as the eluent. Analytical LC was performed with a Gilson HPLC consisting of a pump (model 306), stainless steel column packed with silica gel (Spherisorb $\mathrm{SiO}_{2}, 10 \times 300$ or $4.6 \times 250 \mathrm{~mm})$, differential refractometer (132-RI Detector), UV/vis detector (model 118). EtOAc-petroleum ether (bp $60-90{ }^{\circ} \mathrm{C}$ ) was used as the eluent at a flow rate of $1-4 \mathrm{~mL} / \mathrm{min}$. Solutions were concentrated at a temperature $<60{ }^{\circ} \mathrm{C}$ under diminished pressure.

\subsection{Allyl 3- $O$-acetyl-4,6- $O$-benzylidene- $\alpha$-D- mannopyranoside (2)}

Compound 1 (3.08 g, $10 \mathrm{mmol}$ ) was dissolved in dry $\mathrm{CH}_{2} \mathrm{Cl}_{2}(40 \mathrm{~mL})$ containing $\mathrm{Py}(8.1 \mathrm{~mL}, 100 \mathrm{mmol})$, then under $\mathrm{N}_{2}$ protection, acetyl chloride $(0.8 \mathrm{~mL}, 11$ $\mathrm{mmol})$ in anhyd $\mathrm{CH}_{2} \mathrm{Cl}_{2}(10 \mathrm{~mL})$ was added dropwise to the solution within $30 \mathrm{~min}$ at $0{ }^{\circ} \mathrm{C}$. The reaction mixture was slowly raised to room temperature (rt) and stirred for $2 \mathrm{~h}$, at the end of which time TLC (3:1 petroleum ether-EtOAc) indicated that the reaction was complete. The reaction mixture was diluted with $\mathrm{CH}_{2} \mathrm{Cl}_{2}(100 \mathrm{~mL})$, washed with water, $1 \mathrm{~N} \mathrm{HCl}$, and dried over $\mathrm{Na}_{2} \mathrm{SO}_{4}$. The solution was concentrated, and purification of the residue by column chromatography on a silica gel column (3:1 petroleum ether-EtOAc) gave compound $2(3.17 \mathrm{~g}, 90.6 \%)$ as a syrup: $[\alpha]_{\mathrm{D}}-58.7^{\circ}\left(c 1.0, \mathrm{CHCl}_{3}\right)$; ${ }^{1} \mathrm{H}$ NMR $\left(400 \mathrm{MHz}, \mathrm{CDCl}_{3}\right): \delta 7.46-7.34(\mathrm{~m}, 5 \mathrm{H}$, $\mathrm{PhH}), 5.88\left(\mathrm{~m}, 1 \mathrm{H}, \mathrm{CH}_{2}=\mathrm{CHCH}_{2} \mathrm{O}\right), 5.55(\mathrm{~s}, 1 \mathrm{H}$, $\left.\mathrm{PhCHO}_{2}\right), 5.36\left(\mathrm{dd}, 1 \mathrm{H}, J_{2,3} 3.2 \mathrm{~Hz}, J_{3,4} 10.0 \mathrm{~Hz}, \mathrm{H}-3\right)$, $5.20\left(\mathrm{~m}, 1 \mathrm{H}, \mathrm{CH}_{2}=\mathrm{CHCH}_{2} \mathrm{O}\right), 5.23\left(\mathrm{~m}, 1 \mathrm{H}, \mathrm{CH}_{2}=\right.$ $\left.\mathrm{CHCH}_{2} \mathrm{O}\right), 4.89$ (d, $\left.1 \mathrm{H}, J_{1,2} 1.5 \mathrm{~Hz}, \mathrm{H}-1\right), 4.28$ (dd, 1 $\mathrm{H}, J 4.8,10.6 \mathrm{~Hz}, \mathrm{H}-6 \mathrm{a}), 4.19\left(\mathrm{~m}, 1 \mathrm{H}, \mathrm{CH}_{2}=\mathrm{CHCH}_{2} \mathrm{O}\right)$, 4.15 (dd, $\left.1 \mathrm{H}, J_{1,2} 1.5 \mathrm{~Hz}, J_{2,3} 3.2 \mathrm{~Hz}, \mathrm{H}-2\right), 4.09$ (dd, 1 $\mathrm{H}, J 10.0,10.6 \mathrm{~Hz}, \mathrm{H}-6 \mathrm{~b}), 4.02\left(\mathrm{~m}, 1 \mathrm{H}, \mathrm{CH}_{2}=\right.$ $\mathrm{CHCH}_{2} \mathrm{O}$ ), 3.99 (ddd, $1 \mathrm{H}, J$ 4.8, 10.0, $10.6 \mathrm{~Hz}, \mathrm{H}-5$ ), $3.84\left(\mathrm{dd}, 1 \mathrm{H}, J_{3,4}=J_{4,5}=10.0 \mathrm{~Hz}, \mathrm{H}-4\right), 2.12(\mathrm{~s}, 3 \mathrm{H}$, $\mathrm{CH}_{3} \mathrm{CO}$ ). Anal. Calcd for $\mathrm{C}_{18} \mathrm{H}_{22} \mathrm{O}_{7}: \mathrm{C}, 61.70 ; \mathrm{H}, 6.33$. Found: C, 61.54; H, 6.24.

\subsection{Allyl 3-O-acetyl-4,6-O-benzylidene-2- $O$ - chloroacetyl- $\alpha$-D-mannopyranoside (3)}

Compound 2 (3.50 g, $10 \mathrm{mmol})$ was dissolved in dry $\mathrm{CH}_{2} \mathrm{Cl}_{2}(40 \mathrm{~mL})$ containing $\mathrm{Py}(8.1 \mathrm{~mL}, 100 \mathrm{mmol})$, 
then under $\mathrm{N}_{2}$ protection, chloroacetyl chloride $(0.9 \mathrm{~mL}$, $11 \mathrm{mmol})$ in anhyd $\mathrm{CH}_{2} \mathrm{Cl}_{2}(10 \mathrm{~mL})$ was added dropwise to the solution within $30 \mathrm{~min}$ at $0{ }^{\circ} \mathrm{C}$. The reaction mixture was slowly raised to rt and stirred for $2 \mathrm{~h}$, at the end of which time TLC (3:1 petroleum ether-EtOAc) indicated that the reaction was complete. The reaction mixture was diluted with $\mathrm{CH}_{2} \mathrm{Cl}_{2}(100 \mathrm{~mL})$, washed with water, $1 \mathrm{~N} \mathrm{HCl}$, and dried over $\mathrm{Na}_{2} \mathrm{SO}_{4}$. The solution was concentrated, and purification of the residue by column chromatography on a silica gel column (3:1 petroleum ether-EtOAc) gave compound 3 (3.88 g, $91.1 \%)$ as a syrup: $[\alpha]_{\mathrm{D}}+29.8^{\circ}\left(c 1.0, \mathrm{CHCl}_{3}\right){ }^{1} \mathrm{H} \mathrm{NMR}$ $\left(400 \mathrm{MHz}, \mathrm{CDCl}_{3}\right): \delta$ 7.47-7.35 (m, $\left.5 \mathrm{H}, \mathrm{Ph} H\right), 5.91(\mathrm{~m}$, $\left.1 \mathrm{H}, \mathrm{CH}_{2}=\mathrm{CHCH}_{2} \mathrm{O}\right), 5.58\left(\mathrm{~s}, 1 \mathrm{H}, \mathrm{PhCHO}_{2}\right), 5.36$ (dd, $\left.1 \mathrm{H}, J_{2,3} 3.5 \mathrm{~Hz}, J_{3,4} 9.8 \mathrm{~Hz}, \mathrm{H}-3\right), 5.42\left(\mathrm{dd}, 1 \mathrm{H}, J_{1,2} 1.5\right.$ $\left.\mathrm{Hz}, J_{2,3} 3.5 \mathrm{~Hz}, \mathrm{H}-2\right), 5.34\left(\mathrm{~m}, 1 \mathrm{H}, \mathrm{CH}_{2}=\mathrm{CHCH}_{2} \mathrm{O}\right)$, $5.27\left(\mathrm{~m}, 1 \mathrm{H}, \mathrm{CH}_{2}=\mathrm{CHCH}_{2} \mathrm{O}\right), 4.85\left(\mathrm{~d}, 1 \mathrm{H}, J_{1,2} 1.5 \mathrm{~Hz}\right.$, $\mathrm{H}-1), 4.30$ (dd, $1 \mathrm{H}, J 4.2,10.5 \mathrm{~Hz}, \mathrm{H}-6 \mathrm{a}), 4.23(\mathrm{~m}, 1 \mathrm{H}$, $\left.\mathrm{CH}_{2}=\mathrm{CHCH}_{2} \mathrm{O}\right), 4.18,4.16(\mathrm{ABq}, 2 \mathrm{H}, J 15.2 \mathrm{~Hz}$, $\mathrm{ClCH}_{2} \mathrm{COO}$ ), $4.06-3.98(\mathrm{~m}, 3 \mathrm{H}), 3.84$ (dd, $1 \mathrm{H}, J_{3,4}=$ $\left.J_{4,5}=9.8 \mathrm{~Hz}, \mathrm{H}-4\right), 2.03\left(\mathrm{~s}, 3 \mathrm{H}, \mathrm{CH}_{3} \mathrm{CO}\right)$. Anal. Calcd for $\mathrm{C}_{20} \mathrm{H}_{23} \mathrm{ClO}_{8}$ : C, 56.27; H, 5.43. Found: C, 56.50; H, 5.31 .

\subsection{Allyl 3-O-acetyl-2- $O$-chloroacetyl- $\alpha-\mathrm{D}-$ mannopyranoside (4)}

Compound 3 (4.26 g, $10 \mathrm{mmol}$ ) was dissolved in 90\% TFA $(50 \mathrm{~mL})$, and the mixture was stirred for $2 \mathrm{~h}$ at $\mathrm{rt}$, at the end of which time TLC (2:1 petroleum etherEtOAc) indicated that the reaction was complete. The mixture was diluted with toluene $(200 \mathrm{~mL})$ and concentrated in vacuo directly. The residue was passed through a short silica gel column with 1:1 petroleum ether-EtOAc as the eluent to give $4(3.02 \mathrm{~g}, 89.1 \%)$ as a foamy solid: $[\alpha]_{\mathrm{D}}-93.5^{\circ}\left(c\right.$ 1.0, $\left.\mathrm{CHCl}_{3}\right) ;{ }^{1} \mathrm{H}$ NMR (400 $\left.\mathrm{MHz}, \mathrm{CDCl}_{3}\right): \delta 5.87\left(\mathrm{~m}, 1 \mathrm{H}, \mathrm{CH}_{2}=\mathrm{CHCH}_{2} \mathrm{O}\right), 5.30$ $\left(\mathrm{m}, 1 \mathrm{H}, \mathrm{CH}_{2}=\mathrm{CHCH}_{2} \mathrm{O}\right), 5.29\left(\mathrm{dd}, 1 \mathrm{H}, J_{1,2} 1.2 \mathrm{~Hz}, J_{2,3}\right.$ $3.1 \mathrm{~Hz}, \mathrm{H}-2), 5.24\left(\mathrm{~m}, 1 \mathrm{H}, \mathrm{CH}_{2}=\mathrm{CHCH}_{2} \mathrm{O}\right), 5.22(\mathrm{dd}, 1$ $\left.\mathrm{H}, J_{2,3} 3.1 \mathrm{~Hz}, J_{3,4} 9.8 \mathrm{~Hz}, \mathrm{H}-3\right), 4.85\left(\mathrm{~d}, 1 \mathrm{H}, J_{1,2} 1.2 \mathrm{~Hz}\right.$, $\mathrm{H}-1), 4.20\left(\mathrm{~m}, 1 \mathrm{H}, \mathrm{CH}_{2}=\mathrm{CHCH}_{2} \mathrm{O}\right), 4.13,4.12(\mathrm{ABq}, 2$ $\left.\mathrm{H}, J 15.2 \mathrm{~Hz}, \mathrm{ClCH}_{2} \mathrm{COO}\right), 4.03-3.97(\mathrm{~m}, 2 \mathrm{H}), 3.89-$ $3.87(\mathrm{~m}, 2 \mathrm{H}), 3.76(\mathrm{~m}, 1 \mathrm{H}), 2.07\left(\mathrm{~s}, 3 \mathrm{H}, \mathrm{CH}_{3} \mathrm{CO}\right)$. Anal. Calcd for $\mathrm{C}_{13} \mathrm{H}_{19} \mathrm{ClO}_{8}$ : C, 46.09; H, 5.65. Found: C, 46.30; H, 5.38.

\subsection{Allyl 2,3,4,6-tetra- $O$-benzoyl- $\alpha$-D-mannopyranosyl- $(1 \rightarrow 2)$-3- $O$-acetyl-4,6- $O$-benzylidene- $\alpha$-D-mannopyran- oside (5)}

To a cooled solution $\left(-20{ }^{\circ} \mathrm{C}\right)$ of $2(3.50 \mathrm{~g}, 10 \mathrm{mmol})$ and 2,3,4,6-tetra- $O$-benzoyl- $\alpha$-D-mannopyranosyl trichloroacetimidate $(8.14 \mathrm{~g}, 11 \mathrm{mmol})$ in anhyd $\mathrm{CH}_{2} \mathrm{Cl}_{2}$ $(50 \mathrm{~mL})$ was added TMSOTf $(18 \mu \mathrm{L}, 0.1 \mathrm{mmol})$. The mixture was stirred at this temperature for $2 \mathrm{~h}$ and then quenched with $\mathrm{Et}_{3} \mathrm{~N}$ (2 drops). The solvents were evaporated in vacuo to give a residue that was purified by silica gel column chromatography (2:1 petroleum ether-EtOAc) to give disaccharide $\mathbf{5}(7.53 \mathrm{~g}, 81.1 \%)$ as a syrup: $[\alpha]_{\mathrm{D}}-39.5^{\circ}\left(c 1.0, \mathrm{CHCl}_{3}\right) ;{ }^{1} \mathrm{H}$ NMR $(400 \mathrm{MHz}$, $\left.\mathrm{CDCl}_{3}\right): \delta 8.08-7.29(\mathrm{~m}, 25 \mathrm{H}, \mathrm{PhH}), 6.14(\mathrm{dd}, 1 \mathrm{H}$, $\left.J_{3,4}=J_{4,5}=10.0 \mathrm{~Hz}, \mathrm{H}-4^{\prime}\right), 5.97\left(\mathrm{dd}, 1 \mathrm{H}, J_{2,3} 3.3 \mathrm{~Hz}\right.$, $\left.J_{3,4} 10.0 \mathrm{~Hz}, \mathrm{H}-3^{\prime}\right), 5.84\left(\mathrm{~m}, 1 \mathrm{H}, \mathrm{CH}_{2}=\mathrm{CHCH}_{2} \mathrm{O}\right), 5.29$ $\left(\mathrm{dd}, 1 \mathrm{H}, J_{1,2} 1.5 \mathrm{~Hz}, J_{2,3} 3.3 \mathrm{~Hz}, \mathrm{H}-2^{\prime}\right), 5.71(\mathrm{~s}, 1 \mathrm{H}$, $\mathrm{PhCHO}_{2}$ ), 5.42 (dd, $1 \mathrm{H}, J_{2,3} 3.0 \mathrm{~Hz}, J_{3,4} 9.8 \mathrm{~Hz}, \mathrm{H}-3$ ), $5.29\left(\mathrm{~m}, 1 \mathrm{H}, \mathrm{CH}_{2}=\mathrm{CHCH}_{2} \mathrm{O}\right), 5.21\left(\mathrm{~d}, 1 \mathrm{H}, J_{1,2} 1.5 \mathrm{~Hz}\right.$, $\left.\mathrm{H}-1^{\prime}\right), 5.19\left(\mathrm{~m}, 1 \mathrm{H}, \mathrm{CH}_{2}=\mathrm{CHCH}_{2} \mathrm{O}\right), 5.01\left(\mathrm{~d}, 1 \mathrm{H}, J_{1,2}\right.$ $1.4 \mathrm{~Hz}, \mathrm{H}-1), 2.17$ (s, $3 \mathrm{H}, \mathrm{CH}_{3} \mathrm{CO}$ ). Anal. Calcd for $\mathrm{C}_{52} \mathrm{H}_{48} \mathrm{O}_{16}$ : C, 67.23; H, 5.21. Found: $\mathrm{C}, 67.08 ; \mathrm{H}, 5.50$.

\subsection{Allyl 2,3,4,6-tetra- $O$-benzoyl- $\alpha$-D-mannopyranosyl- $(1 \rightarrow 2)-3-O$-acetyl- $\alpha$-D-mannopyranoside (6)}

Compound 5 (4.64 g, $5 \mathrm{mmol}$ ) was dissolved in 90\% TFA $(50 \mathrm{~mL})$, and the mixture was stirred for $2 \mathrm{~h}$ at $\mathrm{rt}$, at the end of which time TLC (2:1 petroleum etherEtOAc) indicated that the reaction was complete. The mixture was diluted with toluene $(200 \mathrm{~mL})$ and concentrated in vacuo directly. The residue was passed through a short silica gel column with 2:1 petroleum ether-EtOAc as the eluent to give $6(3.59 \mathrm{~g}, 85.5 \%)$ as a foamy solid: $[\alpha]_{\mathrm{D}}-55.0^{\circ}\left(c\right.$ 1.0, $\left.\mathrm{CHCl}_{3}\right) ;{ }^{1} \mathrm{H}$ NMR (400 $\mathrm{MHz}, \mathrm{CDCl}_{3}$ ): $\delta 8.08-7.28(\mathrm{~m}, 20 \mathrm{H}, \mathrm{Ph} H), 5.97$ (dd, 1 $\left.\mathrm{H}, J_{3,4}=J_{4,5}=9.8 \mathrm{~Hz}, \mathrm{H}-4^{\prime}\right), 5.91\left(\mathrm{dd}, 1 \mathrm{H}, J_{2,3} 3.2 \mathrm{~Hz}\right.$, $\left.J_{3,4} 9.8 \mathrm{~Hz}, \mathrm{H}-3^{\prime}\right), 5.85\left(\mathrm{~m}, 1 \mathrm{H}, \mathrm{CH}_{2}=\mathrm{CHCH}_{2} \mathrm{O}\right), 5.70$ (dd, $\left.1 \mathrm{H}, J_{1,2} 1.5 \mathrm{~Hz}, J_{2,3} 3.2 \mathrm{~Hz}, \mathrm{H}-2^{\prime}\right), 5.22\left(\mathrm{~d}, 1 \mathrm{H}, J_{1,2}\right.$ $\left.1.5 \mathrm{~Hz}, \mathrm{H}-1^{\prime}\right), 5.21\left(\mathrm{dd}, 1 \mathrm{H}, J_{2,3} 3.1 \mathrm{~Hz}, J_{3,4} 9.8 \mathrm{~Hz}, \mathrm{H}-\right.$ 3), 5.17-5.10 (m, $\left.2 \mathrm{H}, \mathrm{CH}_{2}=\mathrm{CHCH}_{2} \mathrm{O}\right), 5.03(\mathrm{~d}, 1 \mathrm{H}$, $\left.J_{1,2} 1.6 \mathrm{~Hz}, \mathrm{H}-1\right), 2.22\left(\mathrm{~s}, 3 \mathrm{H}, \mathrm{CH}_{3} \mathrm{CO}\right)$. Anal. Calcd for $\mathrm{C}_{45} \mathrm{H}_{44} \mathrm{O}_{16}$ : C, 64.28; H, 5.27. Found: C, 64.44; H, 5.20.

\subsection{Allyl 2,3,4,6-tetra- $O$-benzoyl- $\alpha$-D-mannopyranosyl- $(1 \rightarrow 3)-4,6-O$-benzylidene- $\alpha$-D-mannopyranoside (7)}

To a cooled solution $\left(-20^{\circ} \mathrm{C}\right)$ of $1(3.08 \mathrm{~g}, 10 \mathrm{mmol})$ and 2,3,4,6-tetra- $O$-benzoyl- $\alpha$-D-mannopyranosyl trichlroacetimidate $(8.14 \mathrm{~g}, 11 \mathrm{mmol})$ in anhyd $\mathrm{CH}_{2} \mathrm{Cl}_{2}$ $(50 \mathrm{~mL})$ was added TMSOTf $(18 \mu \mathrm{L}, 0.05 \mathrm{mmol})$. The mixture was stirred at this temperature for $2 \mathrm{~h}$ and then quenched with $\mathrm{Et}_{3} \mathrm{~N}$ (2 drops). The solvents were evaporated in vacuo to give a residue that was purified by silica gel column chromatography (2:1 petroleum ether-EtOAc) to give disaccharide 7 as a syrup $(6.76 \mathrm{~g}$, $76.3 \%):[\alpha]_{\mathrm{D}}-33.7^{\circ}\left(c \quad 1.0, \mathrm{CHCl}_{3}\right) ;{ }^{1} \mathrm{H}$ NMR $(400$ $\left.\mathrm{MHz}, \mathrm{CDCl}_{3}\right): \delta 8.08-7.19(\mathrm{~m}, 25 \mathrm{H}, \mathrm{Ph} H), 6.08(\mathrm{dd}, 1$ $\left.\mathrm{H}, J_{3,4}=J_{4,5}=10.0 \mathrm{~Hz}, \mathrm{H}-4^{\prime}\right), 5.98\left(\mathrm{dd}, 1 \mathrm{H}, J_{2,3} 3.3 \mathrm{~Hz}\right.$, $\left.J_{3,4} 10.0 \mathrm{~Hz}, \mathrm{H}-3^{\prime}\right), 5.88\left(\mathrm{dd}, 1 \mathrm{H}, J_{1,2} 0.6 \mathrm{~Hz}, J_{2,3} 3.3 \mathrm{~Hz}\right.$, $\left.\mathrm{H}-2^{\prime}\right), 5.82\left(\mathrm{~m}, 1 \mathrm{H}, \mathrm{CH}_{2}=\mathrm{CHCH}_{2} \mathrm{O}\right), 5.67(\mathrm{~s}, 1 \mathrm{H}$, $\left.\mathrm{PhCHO}_{2}\right), 5.62\left(\mathrm{~d}, 1 \mathrm{H}, J_{1,2} 0.6 \mathrm{~Hz}, \mathrm{H}-1^{\prime}\right), 5.29(\mathrm{~m}, 1 \mathrm{H}$, 
$\left.\mathrm{CH}_{2}=\mathrm{CHCH}_{2} \mathrm{O}\right), 5.18\left(\mathrm{~m}, 1 \mathrm{H}, \mathrm{CH}_{2}=\mathrm{CHCH}_{2} \mathrm{O}\right), 4.92$ (d, $\left.1 \mathrm{H}, J_{1,2} 0.8 \mathrm{~Hz}, \mathrm{H}-1\right)$. Anal. Calcd for $\mathrm{C}_{50} \mathrm{H}_{46} \mathrm{O}_{15}: \mathrm{C}$, 67.71; H, 5.23. Found: C, 67.49; H, 5.40.

\subsection{Allyl 2,3,4,6-tetra- $O$-benzoyl- $\alpha$-D-mannopyranosyl- $(1 \rightarrow 3)$-2- $O$-benzoyl-4,6- $O$-benzylidene- $\alpha$-D-mannopyran- oside (8)}

To a solution of 7 (4.43 g, $5.0 \mathrm{mmol})$ in Py $(20 \mathrm{~mL})$ was added benzoyl chloride $(1.2 \mathrm{~mL}, 10 \mathrm{mmol})$. The reaction mixture was stirred at $\mathrm{rt}$ for $12 \mathrm{~h}$ and then quenched with $\mathrm{MeOH}(2.0 \mathrm{~mL})$. The solvents were evaporated and coevaporated with toluene in vacuo to give a residue that was purified by silica gel column chromatography (2:1 petroleum ether-EtOAc) to give disaccharide 8 as a syrup $(4.36 \mathrm{~g}, 88.1 \%):[\alpha]_{\mathrm{D}}-63.3^{\circ}\left(c 1.0, \mathrm{CHCl}_{3}\right) ;{ }^{1} \mathrm{H}$ NMR (400 MHz, $\left.\mathrm{CDCl}_{3}\right): \delta 8.10-7.21(\mathrm{~m}, 30 \mathrm{H}, \mathrm{PhH})$, $6.06\left(\mathrm{dd}, 1 \mathrm{H}, J_{3,4}=J_{4,5}=9.9 \mathrm{~Hz}, \mathrm{H}-4^{\prime}\right), 5.85(\mathrm{~m}, 1 \mathrm{H}$, $\left.\mathrm{CH}_{2}=\mathrm{CHCH}_{2} \mathrm{O}\right), 5.76\left(\mathrm{dd}, 1 \mathrm{H}, J_{1,2} 0.9 \mathrm{~Hz}, J_{2,3} 2.8 \mathrm{~Hz}\right.$, H-2), $5.73\left(\mathrm{dd}, 1 \mathrm{H}, J_{2,3} 3.2 \mathrm{~Hz}, J_{3,4} 9.9 \mathrm{~Hz}, \mathrm{H}-3^{\prime}\right), 5.71$ $\left(\mathrm{s}, 1 \mathrm{H}, \mathrm{PhCHO}_{2}\right), 5.63\left(\mathrm{~d}, 1 \mathrm{H}, J_{1,2} 1.4 \mathrm{~Hz}, J_{2,3} 3.2 \mathrm{~Hz}\right.$, $\left.\mathrm{H}-2^{\prime}\right), 5.48\left(\mathrm{~d}, 1 \mathrm{H}, J_{1,2} 1.4 \mathrm{~Hz}, \mathrm{H}-1^{\prime}\right), 5.30(\mathrm{~m}, 1 \mathrm{H}$, $\left.\mathrm{CH}_{2}=\mathrm{CHCH}_{2} \mathrm{O}\right), 5.22\left(\mathrm{~m}, 1 \mathrm{H}, \mathrm{CH}_{2}=\mathrm{CHCH}_{2} \mathrm{O}\right), 5.04$ (d, $\left.1 \mathrm{H}, J_{1,2} 1.0 \mathrm{~Hz}, \mathrm{H}-1\right)$. Anal. Calcd for $\mathrm{C}_{57} \mathrm{H}_{50} \mathrm{O}_{16}: \mathrm{C}$, 69.08; H, 5.09. Found: C, 68.94; H, 5.22.

\subsection{Allyl $2,3,4,6$-tetra- $O$-benzoyl- $\alpha$-D-mannopyranosyl- $(1 \rightarrow 3)-4,6$-di- $O$-acetyl-2- $O$-benzoyl- $\alpha$-D-mannopyran- oside (10)}

Compound 8 (4.45 g, $5 \mathrm{mmol}$ ) was dissolved in $90 \%$ TFA $(50 \mathrm{~mL})$, and the mixture was stirred for $2 \mathrm{~h}$ at $\mathrm{rt}$, at the end of which time TLC (2:1 petroleum etherEtOAc) indicated that the reaction was complete. The mixture was diluted with toluene $(200 \mathrm{~mL})$ and concentrated in vacuo directly. The residue was passed through a short silica gel column with 2:1 petroleum ether-EtOAc as the eluent to give $\mathbf{9}$ as a foamy solid. Compound 9 was dissolved in $\mathrm{Py}(50 \mathrm{~mL})$, then $\mathrm{Ac}_{2} \mathrm{O}$ $(20 \mathrm{~mL}, 20 \mathrm{mmol})$ was added. The reaction mixture was stirred at $\mathrm{rt}$ for $12 \mathrm{~h}$, at the end of which time TLC (4:1 petroleum ether-EtOAc) indicated that the reaction was complete. The reaction mixture was concentrated, and then the residue was purified by flash column chromatography on a silica gel column $(2: 1$ petroleum etherEtOAc) to give compound $\mathbf{1 0}(4.06 \mathrm{~g}, 82.4 \%$ for two steps) as a foamy solid: $[\alpha]_{\mathrm{D}}-51.6^{\circ}\left(c 1.0, \mathrm{CHCl}_{3}\right) ;{ }^{1} \mathrm{H}$ NMR (400 MHz, $\left.\mathrm{CDCl}_{3}\right): \delta 8.06-7.22(\mathrm{~m}, 25 \mathrm{H}, \mathrm{Ph} H)$, $6.07\left(\mathrm{dd}, 1 \mathrm{H}, J_{3,4}=J_{4,5}=9.8 \mathrm{~Hz}, \mathrm{H}-4^{\prime}\right), 5.84(\mathrm{~m}, 1 \mathrm{H}$, $\left.\mathrm{CH}_{2}=\mathrm{CHCH}_{2} \mathrm{O}\right), 5.68\left(\mathrm{dd}, 1 \mathrm{H}, J_{2,3} 3.0 \mathrm{~Hz}, J_{3,4} 9.8 \mathrm{~Hz}\right.$, $\left.\mathrm{H}-3^{\prime}\right), 5.61\left(\mathrm{dd}, 1 \mathrm{H}, J_{3,4}=J_{4,5}=9.9 \mathrm{~Hz}, \mathrm{H}-4\right), 5.58(\mathrm{~d}, 1$ $\left.\mathrm{H}, J_{1,2} 0.6 \mathrm{~Hz}, J_{2,3} 3.0 \mathrm{~Hz}, \mathrm{H}-2^{\prime}\right), 5.48\left(\mathrm{~d}, 1 \mathrm{H}, J_{1,2} 1.0\right.$ $\left.\mathrm{Hz}, J_{2,3} 3.1 \mathrm{~Hz}, \mathrm{H}-2\right), 5.31\left(\mathrm{~d}, 1 \mathrm{H}, J_{1,2} 0.6 \mathrm{~Hz}, \mathrm{H}-1^{\prime}\right)$, $5.27\left(\mathrm{~m}, 1 \mathrm{H}, \mathrm{CH}_{2}=\mathrm{CHCH}_{2} \mathrm{O}\right), 5.18\left(\mathrm{~m}, 1 \mathrm{H}, \mathrm{CH}_{2}=\right.$
$\left.\mathrm{CHCH}_{2} \mathrm{O}\right), 5.06\left(\mathrm{~d}, 1 \mathrm{H}, J_{1,2} 1.0 \mathrm{~Hz}, \mathrm{H}-1\right), 2.25(\mathrm{~s}, 3 \mathrm{H}$, $\left.\mathrm{CH}_{3} \mathrm{CO}\right), 2.17$ (s, $3 \mathrm{H}, \mathrm{CH}_{3} \mathrm{CO}$ ). Anal. Calcd for $\mathrm{C}_{54} \mathrm{H}_{50} \mathrm{O}_{18}: \mathrm{C}, 65.71 ; \mathrm{H}, 5.11$. Found: $\mathrm{C}, 65.90 ; \mathrm{H}, 5.00$.

\subsection{0. $2,3,4,6$-Tetra- $O$-benzoyl- $\alpha$-D-mannopyranosyl- $(1 \rightarrow 3)-4,6$-di- $O$-acetyl-2- $O$-benzoyl- $\alpha$-D-mannopyran- osyl trichloroacetimidate (11)}

To a solution of $\mathbf{1 0}(4.93 \mathrm{~g}, 5 \mathrm{mmol})$ in $90 \%$ HOAc (50 $\mathrm{mL})$ containing AcONa (1.47 $\mathrm{g}, 15 \mathrm{mmol})$ was added $\mathrm{PdCl}_{2}{ }^{9}$ (270 mg, $2.5 \mathrm{mmol}$ ), and the mixture was stirred for $12 \mathrm{~h}$, at the end of which time TLC (3:1 petroleum ether-EtOAc) indicated that the reaction was complete. The mixture was diluted with $\mathrm{CH}_{2} \mathrm{Cl}_{2}(150 \mathrm{~mL})$, washed with water and satd aq $\mathrm{NaHCO}_{3}$. The organic layer was concentrated, and the residue was passed through a short silica gel column with $2: 1$ petroleum ether-EtOAc as the eluent to give crude 2,3,4,6-tetra- $O$-benzoyl- $\alpha$-Dmannopyranosyl-( $1 \rightarrow 3)-4,6$-di- $O$-acetyl-2- $O$-benzoyl$\alpha, \beta$-D-mannopyranose as a syrup. Dried under high vacuum for $2 \mathrm{~h}$, the syrup was dissolved in $\mathrm{CH}_{2} \mathrm{Cl}_{2}(50$ $\mathrm{mL})$, and $\mathrm{CCl}_{3} \mathrm{CN}(2.5 \mathrm{~mL}, 25 \mathrm{mmol})$ and DBU $(80 \mu \mathrm{L}$, $0.6 \mathrm{mmol})$ were added. ${ }^{10}$ The reaction mixture was stirred for $12 \mathrm{~h}$, at the end of which time TLC (3:1 petroleum ether-EtOAc) indicated that the reaction was complete. Concentration of the reaction mixture, followed by purification of the crude product on a silica gel column with 3:1 petroleum ether-EtOAc as the eluent, furnished the disaccharide donor $11(4.53 \mathrm{~g}, 83.1 \%)$ as a foamy solid: $[\alpha]_{\mathrm{D}}-38.6^{\circ}\left(c 1.0, \mathrm{CHCl}_{3}\right) ;{ }^{1} \mathrm{H}$ NMR (400 $\left.\mathrm{MHz}, \mathrm{CDCl}_{3}\right): \delta 8.68\left(\mathrm{~s}, 1 \mathrm{H}, \mathrm{CN} H \mathrm{CCl}_{3}\right), 8.26-7.25(\mathrm{~m}$, $25 \mathrm{H}, \mathrm{Ph} H), 6.52\left(\mathrm{~d}, 1 \mathrm{H}, J_{1,2} 1.0 \mathrm{~Hz}, \mathrm{H}-1\right), 6.14(\mathrm{dd}, 1$ $\left.\mathrm{H}, J_{3,4}=J_{4,5}=10.1 \mathrm{~Hz}, \mathrm{H}-4^{\prime}\right), 5.77\left(\mathrm{dd}, 1 \mathrm{H}, J_{1,2} 1.8 \mathrm{~Hz}\right.$, $\left.J_{2,3} 3.2 \mathrm{~Hz}, \mathrm{H}-2^{\prime}\right), 5.74\left(\mathrm{dd}, 1 \mathrm{H}, J_{3,4}=J_{4,5}=10.0 \mathrm{~Hz}, \mathrm{H}-\right.$ 4), $5.69\left(\mathrm{dd}, 1 \mathrm{H}, J_{2,3} 3.2 \mathrm{~Hz}, J_{3,4} 10.1 \mathrm{~Hz}, \mathrm{H}-3^{\prime}\right), 5.53(\mathrm{~d}$, $\left.1 \mathrm{H}, J_{1,2} 1.0 \mathrm{~Hz}, J_{2,3} 3.1 \mathrm{~Hz}, \mathrm{H}-2\right), 5.37\left(\mathrm{~d}, 1 \mathrm{H}, J_{1,2} 1.8\right.$ $\left.\mathrm{Hz}, \mathrm{H}_{-1}{ }^{\prime}\right), 2.29$ (s, $3 \mathrm{H}, \mathrm{CH}_{3} \mathrm{CO}$ ), 2.14 (s, $3 \mathrm{H}, \mathrm{CH}_{3} \mathrm{CO}$ ). Anal. Calcd for $\mathrm{C}_{53} \mathrm{H}_{46} \mathrm{Cl}_{3} \mathrm{NO}_{18}$ : C, 58.33; $\mathrm{H}, 4.25$. Found: C, 58.54; H, 4.22.

3.11. Allyl $2,3,4,6$-tetra- $O$-benzoyl- $\alpha$-D-mannopyranosyl$(1 \rightarrow 3)-4,6$-di- $O$-acetyl-2- $O$-benzoyl- $\alpha$-D-mannopyranosyl-( $1 \rightarrow 2)-3,4,6$-tri- $O$-benzoyl- $\alpha$-D-mannopyranoside (13)

To a cooled solution $\left(-20{ }^{\circ} \mathrm{C}\right)$ of $\mathbf{1 2}(1.60 \mathrm{~g}, 3 \mathrm{mmol})$ and $11(3.60 \mathrm{~g}, 3.3 \mathrm{mmol})$ in anhyd $\mathrm{CH}_{2} \mathrm{Cl}_{2}(50 \mathrm{~mL})$ was added TMSOTf $(18 \mu \mathrm{L}, 0.05 \mathrm{mmol})$. The mixture was stirred at this temperature for $2 \mathrm{~h}$, and then quenched with $\mathrm{Et}_{3} \mathrm{~N}$ ( 2 drops). The solvents were evaporated in vacuo to give a residue, which was purified by silica gel column chromatography $(2: 1$ petroleum ether-EtOAc) to give trisaccharide 13 as a syrup $(3.25 \mathrm{~g}, 74.2 \%)$ : $[\alpha]_{\mathrm{D}}$ $-40.5^{\circ}$ ( c 1.0, $\left.\mathrm{CHCl}_{3}\right) ;{ }^{1} \mathrm{H}$ NMR (400 $\left.\mathrm{MHz}, \mathrm{CDCl}_{3}\right): \delta$ 8.14-7.24 (m, $40 \mathrm{H}, \mathrm{Ph} H), 6.17\left(\mathrm{dd}, 1 \mathrm{H}, J_{3,4}=J_{4,5}=\right.$ 
$10.4 \mathrm{~Hz}, \mathrm{H}-4), 5.96\left(\mathrm{~m}, 1 \mathrm{H}, \mathrm{CH}_{2}=\mathrm{CHCH}_{2} \mathrm{O}\right), 5.95$ (dd, $\left.1 \mathrm{H}, J_{3,4}=J_{4,5}=9.9 \mathrm{~Hz}, \mathrm{H}-4\right), 5.83\left(\mathrm{dd}, 1 \mathrm{H}, J_{2,3} 3.2 \mathrm{~Hz}\right.$, $\left.J_{3,4} 10.0 \mathrm{~Hz}, \mathrm{H}-3\right), 5.73\left(\mathrm{~d}, 1 \mathrm{H}, J_{1,2} 1.6 \mathrm{~Hz}, J_{2,3} 3.2 \mathrm{~Hz}\right.$, H-2), $5.68\left(\mathrm{dd}, 1 \mathrm{H}, J_{2,3} 3.2 \mathrm{~Hz}, J_{3,4} 9.9 \mathrm{~Hz}, \mathrm{H}-3\right), 5.61$ $\left(\mathrm{dd}, 1 \mathrm{H}, J_{3,4}=J_{4,5}=9.9 \mathrm{~Hz}, \mathrm{H}-4\right), 5.58\left(\mathrm{~d}, 1 \mathrm{H}, J_{1,2} 1.7\right.$ $\left.\mathrm{Hz}, J_{2,3} 2.9 \mathrm{~Hz}, \mathrm{H}-2\right), 5.37$ (d, $\left.1 \mathrm{H}, J_{1,2} 1.6 \mathrm{~Hz}, \mathrm{H}-1\right), 5.33$ $\left(\mathrm{m}, 1 \mathrm{H}, \mathrm{CH}_{2}=\mathrm{CHCH}_{2} \mathrm{O}\right), 5.26\left(\mathrm{~m}, 1 \mathrm{H}, \mathrm{CH}_{2}=\right.$ $\left.\mathrm{CHCH}_{2} \mathrm{O}\right), 5.22\left(\mathrm{~d}, 1 \mathrm{H}, J_{1,2} 1.6 \mathrm{~Hz}, \mathrm{H}-1\right), 5.17(\mathrm{~d}, 1$ $\left.\mathrm{H}, J_{1,2} 1.4 \mathrm{~Hz}, \mathrm{H}-1\right), 2.21\left(\mathrm{~s}, 3 \mathrm{H}, \mathrm{CH}_{3} \mathrm{CO}\right), 2.13(\mathrm{~s}, 3 \mathrm{H}$, $\left.\mathrm{CH}_{3} \mathrm{CO}\right) ;{ }^{13} \mathrm{C}$ NMR $\left(100 \mathrm{MHz}, \mathrm{CDCl}_{3}\right): \delta 170.6,170.2$ $\left(2 \mathrm{C}, 2 \mathrm{CH}_{3} \mathrm{CO}\right), 166.2,166.0,165.8,165.5,165.5,165.4$, 165.2, $165.1(8 \mathrm{C}, 8 \mathrm{PhCO}), 118.4\left(\mathrm{CH}_{2}=\mathrm{CHCH}_{2} \mathrm{O}\right)$, 99.6, 99.3, 97.8 (3 C, 3 C-1), 77.6, 76.3, 71.4, 71.0, 70.4, 69.9, 69.8, 69.7, 69.1, 68.9, 67.5, 67.2, 66.1, 63.6, 62.7, $62.1\left(16 \mathrm{C}, \mathrm{C}-2-6, \mathrm{CH}_{2}=\mathrm{CHCH}_{2} \mathrm{O}\right), 20.8,20.8(2 \mathrm{C}, 2$ $\mathrm{CH}_{3} \mathrm{CO}$ ). Anal. Calcd for $\mathrm{C}_{81} \mathrm{H}_{72} \mathrm{O}_{26}: \mathrm{C}, 66.57 ; \mathrm{H}, 4.97$. Found: C, 66.74; H, 5.20.

\subsection{2,3,4,6-Tetra- $O$-benzoyl- $\alpha$-D-mannopyranosyl-} $(1 \rightarrow 3)-4,6$-di- $O$-acetyl-2- $O$-benzoyl- $\alpha$-D-mannopyranosyl-( $1 \rightarrow 2)-3,4,6$-tri- $O$-benzoyl- $\alpha$-D-mannopyranosyl trichloroacetimidate (14)

Compound 13 (1.16 g, $0.8 \mathrm{mmol})$ was deallylated and subsequently trichloroacetimidated under the same conditions as those that were used for the preparation of $\mathbf{1 1}$ from $\mathbf{9}$, giving $\mathbf{1 4}(1.07 \mathrm{~g}, 85.6 \%)$ as a foamy solid: $\left.[\alpha]_{\mathrm{D}}-30.5^{\circ}(c) 1.0, \mathrm{CHCl}_{3}\right) ;{ }^{1} \mathrm{H}$ NMR $(400 \mathrm{MHz}$, $\left.\mathrm{CDCl}_{3}\right): \delta 8.66\left(\mathrm{~s}, 1 \mathrm{H}, \mathrm{CNHCCl}{ }_{3}\right), 8.14-7.25(\mathrm{~m}, 40$ $\mathrm{H}, \mathrm{Ph} H), 6.60\left(\mathrm{~d}, 1 \mathrm{H}, J_{1,2} 1.0 \mathrm{~Hz}, \mathrm{H}-1\right), 6.17(\mathrm{dd}, 1 \mathrm{H}$, $\left.J_{3,4}=J_{4,5}=10.0 \mathrm{~Hz}, \mathrm{H}-4\right), 6.08\left(\mathrm{dd}, 1 \mathrm{H}, J_{3,4}=J_{4,5}=\right.$ $9.9 \mathrm{~Hz}, \mathrm{H}-4), 5.85$ (dd, $1 \mathrm{H}, J_{2,3} 3.2 \mathrm{~Hz}, J_{3,4} 10.0 \mathrm{~Hz}, \mathrm{H}-$ 3), $5.72\left(\mathrm{~d}, 1 \mathrm{H}, J_{1,2} 1.5 \mathrm{~Hz}, J_{2,3} 3.3 \mathrm{~Hz}, \mathrm{H}-2\right), 5.68(\mathrm{dd}, 1$ $\left.\mathrm{H}, J_{2,3} 3.3 \mathrm{~Hz}, J_{3,4} 9.9 \mathrm{~Hz}, \mathrm{H}-3\right), 5.62\left(\mathrm{dd}, 1 \mathrm{H}, J_{3,4}=\right.$ $\left.J_{4,5}=9.8 \mathrm{~Hz}, \mathrm{H}-4\right), 5.59\left(\mathrm{~d}, 1 \mathrm{H}, J_{1,2} 1.6 \mathrm{~Hz}, J_{2,3} 3.0 \mathrm{~Hz}\right.$, $\mathrm{H}-2), 5.35\left(\mathrm{~d}, 1 \mathrm{H}, J_{1,2} 1.6 \mathrm{~Hz}, \mathrm{H}-1\right), 5.30\left(\mathrm{~d}, 1 \mathrm{H}, J_{1,2} 1.5\right.$ $\mathrm{Hz}, \mathrm{H}-1), 2.20$ (s, $3 \mathrm{H}, \mathrm{CH}_{3} \mathrm{CO}$ ), 2.11 (s, $3 \mathrm{H}, \mathrm{CH}_{3} \mathrm{CO}$ ). Anal. Calcd for $\mathrm{C}_{80} \mathrm{H}_{68} \mathrm{Cl}_{3} \mathrm{NO}_{26}: \mathrm{C}, 61.37 ; \mathrm{H}, 4.38$. Found: C, 61.50; H, 4.22.

3.13. Allyl 2,3,4,6-tetra- $O$-benzoyl- $\alpha$-D-mannopyranosyl$(1 \rightarrow 2)-3,4,6$-tri- $O$-benzoyl- $\alpha$-D-mannopyranosyl- $(1 \rightarrow 2)$ 3,4,6-tri- $O$-benzoyl- $\alpha$-D-mannopyranosyl-(1 $\rightarrow$ 6)-3- $O$ acetyl-2- $O$-chloroacetyl- $\alpha$-D-mannopyranoside (16)

Compound 4 (676 mg, $2.0 \mathrm{mmol})$ and 15 (3.38 g, 2.0 $\mathrm{mmol}$ ) were coupled under the same conditions as those that were used for the preparation of $\mathbf{1 3}$ from $\mathbf{1 1}$ and $\mathbf{1 2}$, giving 16 as a foamy solid $(3.30 \mathrm{~g}, 88.5 \%)$ : $[\alpha]_{\mathrm{D}}-26.5^{\circ}$ (c 1.0, $\mathrm{CHCl}_{3}$ ); ${ }^{1} \mathrm{H}$ NMR (400 MHz, $\mathrm{CDCl}_{3}$ ): $\delta 8.06-$ $7.15(\mathrm{~m}, 50 \mathrm{H}, \mathrm{Ph} H), 6.05\left(\mathrm{dd}, 1 \mathrm{H}, J_{3,4}=J_{4,5}=9.7 \mathrm{~Hz}\right.$, $\mathrm{H}-4), 5.97\left(\mathrm{dd}, 1 \mathrm{H}, J_{3,4}=J_{4,5}=9.8 \mathrm{~Hz}, \mathrm{H}-4\right), 5.97-5.90$ $(\mathrm{m}, 4 \mathrm{H}), 5.78\left(\mathrm{dd}, 1 \mathrm{H}, J_{2,3} 2.8 \mathrm{~Hz}, J_{3,4} 9.7 \mathrm{~Hz}, \mathrm{H}-3\right)$, $5.77\left(\mathrm{~d}, 1 \mathrm{H}, J_{1,2} 0.8 \mathrm{~Hz}, J_{2,3} 3.1 \mathrm{~Hz}, \mathrm{H}-2\right), 5.41(\mathrm{~d}, 1 \mathrm{H}$, $\left.J_{1,2} 0.8 \mathrm{~Hz}, \mathrm{H}-1\right), 5.77\left(\mathrm{~d}, 1 \mathrm{H}, J_{2,3} 1.0 \mathrm{~Hz}, J_{2,3} 2.9 \mathrm{~Hz}\right.$,
$\mathrm{H}-2), 5.32-5.22\left(\mathrm{~m}, 2 \mathrm{H}, \mathrm{CH}_{2}=\mathrm{CHCH}_{2} \mathrm{O}\right), 5.28(\mathrm{~d}, 1 \mathrm{H}$, $\left.J_{1,2} 1.0 \mathrm{~Hz}, \mathrm{H}-1\right), 4.99$ (d, $\left.1 \mathrm{H}, J_{1,2} 0.8 \mathrm{~Hz}, \mathrm{H}-1\right), 4.93$ (d, $\left.1 \mathrm{H}, J_{1,2} 0.5 \mathrm{~Hz}, \mathrm{H}-1\right), 4.11,4.09(\mathrm{ABq}, 2 \mathrm{H}, J 15.3 \mathrm{~Hz}$, $\left.\mathrm{ClCH}_{2} \mathrm{COO}\right), 2.03\left(\mathrm{~s}, 3 \mathrm{H}, \mathrm{CH}_{3} \mathrm{CO}\right) ;{ }^{13} \mathrm{C}$ NMR $(100$ $\left.\mathrm{MHz}, \mathrm{CDCl}_{3}\right): \delta 171.0\left(\mathrm{CH}_{3} \mathrm{CO}\right), 166.9,166.6,166.5$, $165.9,165.8,165.6,165.5,165.4,165.4,165.1,164.9$ (11 C, $\left.10 \mathrm{PhCO}, \mathrm{ClCH}_{2} \mathrm{CO}\right), 118.4\left(\mathrm{CH}_{2}=\mathrm{CHCH}_{2} \mathrm{O}\right)$, 100.6, 99.7, 98.4, 96.5 (4 C, 4 C-1), 72.3, 72.1, 71.4, 71.1, 70.6, 70.2, 69.8, 69.7, 69.6, 68.7, 68.7, 68.0, 67.5, 66.7, 65.6, 65.6, 65.5, 64.0, 63.7, 62.8, 60.5 (21 C, C-2-6, $\left.\mathrm{CH}_{2}=\mathrm{CHCH}_{2} \mathrm{O}\right), 29.6\left(\mathrm{ClCH}_{2} \mathrm{CO}\right), 20.9\left(\mathrm{CH}_{3} \mathrm{CO}\right)$. Anal. Calcd for $\mathrm{C}_{101} \mathrm{H}_{89} \mathrm{ClO}_{33}$ : C, 65.00; H, 4.81. Found: C, 65.14; H, 5.03.

3.14. Allyl 2,3,4,6-tetra- $O$-benzoyl- $\alpha$-D-mannopyranosyl$(1 \rightarrow 2)-3,4,6$-tri- $O$-benzoyl- $\alpha$-D-mannopyranosyl-( $1 \rightarrow 2)$ 3,4,6-tri- $O$-benzoyl- $\alpha$-D-mannopyranosyl-( $1 \rightarrow 6)$-3,4-di$O$-acetyl-2- $O$-chloroacetyl- $\alpha$-D-mannopyranoside (17)

To a solution of $\mathbf{1 6}(1.85 \mathrm{~g}, 1 \mathrm{mmol})$ in Py $(20 \mathrm{~mL})$ was added $\mathrm{Ac}_{2} \mathrm{O}(10 \mathrm{~mL}, 10 \mathrm{mmol})$. The reaction mixture was stirred at $\mathrm{rt}$ for $12 \mathrm{~h}$, at the end of which time TLC (2:1 petroleum ether-EtOAc) indicated that the reaction was complete. The reaction mixture was concentrated, and then the residue was purified by flash column chromatography on a silica gel column (2:1 petroleum ether-EtOAc) to give compound $17(1.81 \mathrm{~g}, 94.8 \%)$ as a foamy solid: $[\alpha]_{\mathrm{D}}-8.1^{\circ}\left(c 1.0, \mathrm{CHCl}_{3}\right) ;{ }^{1} \mathrm{H}$ NMR $(400$ $\left.\mathrm{MHz}, \mathrm{CDCl}_{3}\right): \delta 8.02-7.11(\mathrm{~m}, 50 \mathrm{H}, \mathrm{Ph} H), 6.08(\mathrm{dd}, 1$ $\left.\mathrm{H}, J_{3,4}=J_{4,5}=9.9 \mathrm{~Hz}, \mathrm{H}-4\right), 6.02\left(\mathrm{dd}, 1 \mathrm{H}, J_{3,4}=J_{4,5}=\right.$ $9.8 \mathrm{~Hz}, \mathrm{H}-4), 6.01\left(\mathrm{dd}, 1 \mathrm{H}, J_{3,4}=J_{4,5}=9.8 \mathrm{~Hz}, \mathrm{H}-4\right)$, 5.95-5.88 (m, 3 H), 5.76-5.73 (m, $2 \mathrm{H}), 5.45(\mathrm{dd}, 1 \mathrm{H}$, $\left.J_{3,4}=J_{4,5}=9.9 \mathrm{~Hz}, \mathrm{H}-4\right), 5.44-5.35(\mathrm{~m}, 2 \mathrm{H}), 5.44(\mathrm{~d}, 1$ $\left.\mathrm{H}, J_{1,2} 0.5 \mathrm{~Hz}, \mathrm{H}-1\right), 5.40\left(\mathrm{~d}, 1 \mathrm{H}, J_{1,2} 1.2 \mathrm{~Hz}, \mathrm{H}-1\right), 5.26$ $\left(\mathrm{m}, 1 \mathrm{H}, \mathrm{CH}_{2}=\mathrm{CHCH}_{2} \mathrm{O}\right), 5.19\left(\mathrm{~d}, 1 \mathrm{H}, J_{1,2} 1.4 \mathrm{~Hz}, \mathrm{H}-\right.$ 1), $4.95\left(\mathrm{~d}, 1 \mathrm{H}, J_{1,2} 1.0 \mathrm{~Hz}, \mathrm{H}-1\right), 4.16,4.07(\mathrm{ABq}, 2 \mathrm{H}$, $\left.J 15.1 \mathrm{~Hz}, \mathrm{ClCH}_{2} \mathrm{COO}\right), 2.04$ (s, $3 \mathrm{H}, \mathrm{CH}_{3} \mathrm{CO}$ ), 2.02 (s, 3 $\left.\mathrm{H}, \mathrm{CH}_{3} \mathrm{CO}\right) ;{ }^{13} \mathrm{C}$ NMR $\left(100 \mathrm{MHz}, \mathrm{CDCl}_{3}\right): \delta$ 170.0, 169.7 ( $\left.2 \mathrm{C}, 2 \mathrm{CH}_{3} \mathrm{CO}\right), 167.0,166.4,166.4,165.9,165.7$, 165.7, 165.5, 165.4, 165.4, 165.1, 164.9 (11 C, $10 \mathrm{PhCO}$, $\left.\mathrm{ClCH}_{2} \mathrm{CO}\right), 118.7\left(\mathrm{CH}_{2}=\mathrm{CHCH}_{2} \mathrm{O}\right), 100.7,99.7$, 98.6, 96.2 (4 C, 4 C-1), 71.5, 71.4, 70.5, 70.2, 69.9, 69.7, 69.6, 69.6, 69.4, 69.0, 68.8, 68.8, 67.6, 67.2, 66.6, 66.2, 66.0, 63.8, 63.8, 63.7, 62.8 (21 C, C-2-6, $\mathrm{CH}_{2}=\mathrm{CHCH}_{2} \mathrm{O}$ ), $29.7\left(\mathrm{ClCH}_{2} \mathrm{CO}\right), 20.9,20.8\left(\mathrm{CH}_{3} \mathrm{CO}\right)$. Anal. Calcd for $\mathrm{C}_{103} \mathrm{H}_{91} \mathrm{ClO}_{34}: \mathrm{C}, 64.83 ; \mathrm{H}, 4.81$. Found: $\mathrm{C}, 64.90 ; \mathrm{H}$, 5.02 .

3.15. Allyl $2,3,4,6$-tetra- $O$-benzoyl- $\alpha$-D-mannopyranosyl$(1 \rightarrow 2)-3,4,6$-tri- $O$-benzoyl- $\alpha$-D-mannopyranosyl-(1 $\rightarrow 2)$ 3,4,6-tri- $O$-benzoyl- $\alpha$-D-mannopyranosyl- $(1 \rightarrow 6)-3,4$-di$O$-acetyl- $\alpha$-D-mannopyranoside (18)

To a solution of $17(1.80 \mathrm{~g}, 0.94 \mathrm{mmol})$ in $\mathrm{EtOH}(25$ $\mathrm{mL})-\mathrm{CH}_{2} \mathrm{Cl}_{2}(100 \mathrm{~mL})$ was added thiourea $(0.36 \mathrm{~g})$, and 
the mixture was refluxed for $16 \mathrm{~h}$, at the end of which time TLC (3:1 petroleum ether-EtOAc) indicated that the reaction was complete. The mixture was concentrated. The residue was passed through a silica gel column with $3: 1$ petroleum ether-EtOAc as the eluent to give $18(1.44 \mathrm{~g}, 83.7 \%)$ as a foamy solid: $[\alpha]_{\mathrm{D}}-16.5^{\circ}$ (c $\left.1.0, \mathrm{CHCl}_{3}\right) ;{ }^{1} \mathrm{H}$ NMR $\left(400 \mathrm{MHz}, \mathrm{CDCl}_{3}\right): \delta 8.09-$ $7.11(\mathrm{~m}, 50 \mathrm{H}, \mathrm{Ph} H), 6.06\left(\mathrm{dd}, 1 \mathrm{H}, J_{3,4}=J_{4,5}=9.8 \mathrm{~Hz}\right.$, $\mathrm{H}-4), 6.02\left(\mathrm{dd}, 1 \mathrm{H}, J_{3,4}=J_{4,5}=9.9 \mathrm{~Hz}, \mathrm{H}-4\right), 5.97-5.85$ (m, $4 \mathrm{H}), 5.76-5.73(\mathrm{~m}, 2 \mathrm{H}), 5.49\left(\mathrm{dd}, 1 \mathrm{H}, J_{3,4}=J_{4,5}=\right.$ $9.9 \mathrm{~Hz}, \mathrm{H}-4), 5.42\left(\mathrm{~d}, 1 \mathrm{H}, J_{1,2} 0.6 \mathrm{~Hz}, \mathrm{H}-1\right), 5.35-5.30$ $(\mathrm{m}, 2 \mathrm{H}), 5.28\left(\mathrm{~d}, 1 \mathrm{H}, J_{1,2} 1.5 \mathrm{~Hz}, \mathrm{H}-1\right), 5.19(\mathrm{~m}, 1 \mathrm{H}$, $\left.\mathrm{CH}_{2}=\mathrm{CHCH}_{2} \mathrm{O}\right), 4.93\left(\mathrm{~d}, 1 \mathrm{H}, J_{1,2} 1.4 \mathrm{~Hz}, \mathrm{H}-1\right), 4.91$ (d, $1 \mathrm{H}, \mathrm{H}-1), 2.09$ (s, $3 \mathrm{H}, \mathrm{CH}_{3} \mathrm{CO}$ ), 2.04 (s, $3 \mathrm{H}, \mathrm{CH}_{3} \mathrm{CO}$ ); ${ }^{13} \mathrm{C}$ NMR $\left(100 \mathrm{MHz}, \mathrm{CDCl}_{3}\right): \delta 170.2,169.8(2 \mathrm{C}, 2$ $\left.\mathrm{CH}_{3} \mathrm{CO}\right), 166.6,166.5,166.1,165.7,165.6,165.5,165.4$, $165.4,165.1,164.8(10 \mathrm{C}, 10 \mathrm{PhCO}), 118.1\left(\mathrm{CH}_{2}=\right.$ $\left.\mathrm{CHCH}_{2} \mathrm{O}\right), 100.2,99.7,98.9,98.7$ (4 C, $\left.4 \mathrm{C}-1\right), 72.0$, $71.2,70.6,70.2,70.0,69.9,69.7,69.5,69.4,68.9,68.5$, $68.5,67.6,67.5,66.6,66.5,66.2,63.9,63.8,62.8,60.5(21$ $\left.\mathrm{C}, \mathrm{C}-2-6, \mathrm{CH}_{2}=\mathrm{CHCH}_{2} \mathrm{O}\right), 21.1,20.9\left(\mathrm{CH}_{3} \mathrm{CO}\right)$. Anal. Calcd for $\mathrm{C}_{101} \mathrm{H}_{90} \mathrm{O}_{33}: \mathrm{C}, 66.22 ; \mathrm{H}, 4.95$. Found: $\mathrm{C}$, 65.94; $\mathrm{H}, 4.83$.

3.16. Allyl 2,3,4,6-tetra- $O$-benzoyl- $\alpha$-D-mannopyranosyl$(1 \rightarrow 2)-3,4,6$-tri- $O$-benzoyl- $\alpha$-D-mannopyranosyl-( $1 \rightarrow 2)$ 3,4,6-tri- $O$-benzoyl- $\alpha$-D-mannopyranosyl-( $(1 \rightarrow 6)-[2,3,4,6-$ tetra- $O$-benzoyl- $\alpha$-D-mannopyranosyl-( $1 \rightarrow 3)-4,6$-di- $O$ acetyl-2- $O$-benzoyl- $\alpha$-D-mannopyranosyl-( $1 \rightarrow 2)-3,4,6-$ tri- $O$-benzoyl- $\alpha$-D-mannopyranosyl- $(1 \rightarrow 2)]-3,4-\mathrm{di}-O$ acetyl- $\alpha$-D-mannopyranoside (19)

Compound 14 (1.31 g, $0.84 \mathrm{mmol})$ and 18 (1.40 g, 0.76 $\mathrm{mmol}$ ) were coupled under the same conditions as that used for the preparation of $\mathbf{1 3}$ from $\mathbf{1 1}$ and $\mathbf{1 2}$, giving $\mathbf{1 9}$ $(1.66 \mathrm{~g}, 67.5 \%)$ as a foamy solid: $[\alpha]_{\mathrm{D}}-31.5^{\circ}(c 1.0$, $\mathrm{CHCl}_{3}$ ); ${ }^{1} \mathrm{H} \mathrm{NMR}\left(400 \mathrm{MHz}, \mathrm{CDCl}_{3}\right.$ ), (some characteristic signals are given): $\delta 6.14\left(\mathrm{dd}, 1 \mathrm{H}, J_{3,4}=J_{4,5}=9.8\right.$ $\mathrm{Hz}, \mathrm{H}-4), 6.03\left(\mathrm{dd}, 1 \mathrm{H}, J_{3,4}=J_{4,5}=9.8 \mathrm{~Hz}, \mathrm{H}-4\right), 5.96$ $\left(\mathrm{m}, 1 \mathrm{H}, \mathrm{OCH}_{2} \mathrm{CH}=\mathrm{CH}_{2}\right), 5.72\left(\mathrm{dd}, 1 \mathrm{H}, J_{1,2}=1.0 \mathrm{~Hz}\right.$, $\left.J_{2,3}=3.1 \mathrm{~Hz}, \mathrm{H}-2\right), 5.68\left(\mathrm{dd}, 1 \mathrm{H}, J_{2,3}=3.1 \mathrm{~Hz}, J_{3,4}=\right.$ $9.7 \mathrm{~Hz}, \mathrm{H}-3), 5.59\left(\mathrm{dd}, 1 \mathrm{H}, J_{3,4}=J_{4,5}=9.9 \mathrm{~Hz}, \mathrm{H}-4\right)$, $5.55\left(\mathrm{dd}, 1 \mathrm{H}, J_{1,2}=1.4 \mathrm{~Hz}, J_{2,3}=3.2 \mathrm{~Hz}, \mathrm{H}-2\right), 5.48(\mathrm{~d}$, $\left.1 \mathrm{H}, J_{1,2} 0.7 \mathrm{~Hz}, \mathrm{H}-1\right), 5.46\left(\mathrm{~d}, 1 \mathrm{H}, J_{1,2} 0.6 \mathrm{~Hz}, \mathrm{H}-1\right)$, $5.37\left(\mathrm{~d}, 1 \mathrm{H}, J_{1,2} 1.9 \mathrm{~Hz}, \mathrm{H}-1\right), 5.33\left(\mathrm{~d}, 1 \mathrm{H}, J_{1,2} 1.9 \mathrm{~Hz}\right.$, $\mathrm{H}-1), 5.23\left(\mathrm{~m}, 1 \mathrm{H}, \mathrm{CH}_{2}=\mathrm{CHCH}_{2} \mathrm{O}\right), 5.15\left(\mathrm{~d}, 1 \mathrm{H}, J_{1,2}\right.$ $0.7 \mathrm{~Hz}, \mathrm{H}-1), 5.06\left(\mathrm{~s}, 1 \mathrm{H}, J_{1,2} 1.7 \mathrm{~Hz}, \mathrm{H}-1\right), 4.92(\mathrm{~d}, 1 \mathrm{H}$, $\left.J_{1,2} 1.5 \mathrm{~Hz}, \mathrm{H}-1\right), 2.19\left(\mathrm{~s}, 3 \mathrm{H}, \mathrm{CH}_{3} \mathrm{CO}\right), 2.12(\mathrm{~s}, 3 \mathrm{H}$, $\mathrm{CH}_{3} \mathrm{CO}$ ), 2.09 (s, $\left.3 \mathrm{H}, \mathrm{CH}_{3} \mathrm{CO}\right), 2.01\left(\mathrm{~s}, 3 \mathrm{H}, \mathrm{CH}_{3} \mathrm{CO}\right.$ ); ${ }^{13} \mathrm{C}$ NMR $\left(100 \mathrm{MHz}, \mathrm{CDCl}_{3}\right): \delta 170.6,170.3,170.2$, 169.7 ( $\left.4 \mathrm{C}, 4 \mathrm{CH}_{3} \mathrm{CO}\right), 166.4,166.3,166.2,165.9,165.8$, 165.7, 165.6, 165.5, 165.4, 164.8 (18 C, $18 \mathrm{PhCO}$, some signals overlapped), $118.2\left(\mathrm{CH}_{2}=\mathrm{CHCH}_{2} \mathrm{O}\right), 100.3$, 100.0, 99.7, 99.4, 99.2, 98.7, 97.9 (7 C, 7 C-1), 21.1, 20.9, 20.9, 20.8 (4 C, $4 \mathrm{CH}_{3} \mathrm{CO}$ ). Anal. Calcd for
$\mathrm{C}_{179} \mathrm{H}_{156} \mathrm{O}_{58}: \mathrm{C}, 66.45 ; \mathrm{H}, 4.86$. Found: $\mathrm{C}, 66.64 ; \mathrm{H}$, 5.05 .

3.17. 2,3,4,6-Tetra- $O$-benzoyl- $\alpha$-D-mannopyranosyl$(1 \rightarrow 2)-3,4,6$-tri- $O$-benzoyl- $\alpha$-D-mannopyranosyl-( $1 \rightarrow 2)$ 3,4,6-tri- $O$-benzoyl- $\alpha$-D-mannopyranosyl-( $1 \rightarrow 6)-[2,3,4,6-$ tetra- $O$-benzoyl- $\alpha$-D-mannopyranosyl- $(1 \rightarrow 3)-4,6-\mathrm{di}-O$ acetyl-2- $O$-benzoyl- $\alpha$-D-mannopyranosyl- $(1 \rightarrow 2)-3,4,6-$ tri- $O$-benzoyl- $\alpha$-D-mannopyranosyl- $(1 \rightarrow 2)]-3,4-\mathrm{di}-O$ acetyl- $\alpha$-D-mannopyranosyl trichloroacetimidate (20)

Compound 19 (1.50 g, $0.46 \mathrm{mmol})$ was deallylated and subsequently trichloroacetimidated under the same conditions as those used for the preparation of $\mathbf{1 1}$ from 10, giving $20(1.21 \mathrm{~g}, 78.1 \%)$ as a foamy solid: $\left.[\alpha]_{\mathrm{D}}-36.3^{\circ}(c) 1.0, \mathrm{CHCl}_{3}\right) ;{ }^{1} \mathrm{H}$ NMR $(400 \mathrm{MHz}$, $\mathrm{CDCl}_{3}$ ), (some characteristic signals are given): $\delta 8.68$ (s, $\left.1 \mathrm{H}, \mathrm{CNHCCL}_{3}\right), 6.47$ (d, $\left.1 \mathrm{H}, J_{1,2} 1.8 \mathrm{~Hz}, \mathrm{H}-1\right), 6.18$ $\left(\mathrm{dd}, 1 \mathrm{H}, J_{3,4}=J_{4,5}=9.9 \mathrm{~Hz}, \mathrm{H}-4\right), 5.73\left(\mathrm{~d}, 1 \mathrm{H}, J_{1,2} 0.7\right.$ $\mathrm{Hz}, \mathrm{H}-1), 5.47$ (d, $\left.1 \mathrm{H}, J_{1,2} 1.0 \mathrm{~Hz}, \mathrm{H}-1\right), 5.36(\mathrm{~d}, 2 \mathrm{H}$, $\left.J_{1,2} 1.0 \mathrm{~Hz}, 2 \mathrm{H}-1\right), 5.14\left(\mathrm{~d}, 1 \mathrm{H}, J_{1,2} 0.7 \mathrm{~Hz}, \mathrm{H}-1\right), 4.91$ (d, $\left.1 \mathrm{H}, J_{1,2} 0.8 \mathrm{~Hz}, \mathrm{H}-1\right), 2.17$ (s, $3 \mathrm{H}, \mathrm{CH}_{3} \mathrm{CO}$ ), 2.16 (s, $3 \mathrm{H}, \mathrm{CH}_{3} \mathrm{CO}$ ), $2.10\left(\mathrm{~s}, 3 \mathrm{H}, \mathrm{CH}_{3} \mathrm{CO}\right), 2.01(\mathrm{~s}, 3 \mathrm{H}$, $\mathrm{CH}_{3} \mathrm{CO}$ ). Anal. Calcd for $\mathrm{C}_{178} \mathrm{H}_{152} \mathrm{Cl}_{3} \mathrm{NO}_{58}$ : C, 64.02; $\mathrm{H}, 4.59$. Found: $\mathrm{C}, 64.30 ; \mathrm{H}, 4.40$.

3.18. Allyl $2,3,4,6$-tetra- $O$-benzoyl- $\alpha$-D-mannopyranosyl$(1 \rightarrow 2)-3,4,6$-tri- $O$-benzoyl- $\alpha$-D-mannopyranosyl-( $1 \rightarrow 2)$ $3,4,6$-tri- $O$-benzoyl- $\alpha$-D-mannopyranosyl-( $1 \rightarrow 6)$-[2,3,4,6tetra- $O$-benzoyl- $\alpha$-D-mannopyranosyl-( $1 \rightarrow 3)-4,6$-di- $O$ acetyl-2- $O$-benzoyl- $\alpha$-D-mannopyranosyl-( $1 \rightarrow 2)-3,4,6-$ tri- $O$-benzoyl- $\alpha$-D-mannopyranosyl-( $(1 \rightarrow 2)]-3,4-d i-O$ acetyl- $\alpha$-D-mannopyranosyl- $(1 \rightarrow 6)-[2,3,4,6$-tetra- $O$ benzoyl- $\alpha$-D-mannopyranosyl-( $1 \rightarrow 2)]-3-O$-acetyl- $\alpha$-Dmannopyranoside (21)

To a cooled solution $\left(-20^{\circ} \mathrm{C}\right)$ of $\mathbf{2 0}(1.00 \mathrm{~g}, 0.3 \mathrm{mmol})$ and $6(0.50 \mathrm{~g}, 0.6 \mathrm{mmol})$ in anhyd $\mathrm{CH}_{2} \mathrm{Cl}_{2}(50 \mathrm{~mL})$ was added TMSOTf $(9 \mu \mathrm{L}, 0.05 \mathrm{mmol})$. The mixture was stirred at this temperature for $2 \mathrm{~h}$ and then quenched with $\mathrm{Et}_{3} \mathrm{~N}$ (2 drops). The solvents were evaporated in vacuo to give a residue that was purified by silica gel column chromatography (2:1 petroleum ether-EtOAc) to give nonasaccharide 21 (898 $\mathrm{mg}$, $74.8 \%)$ as a syrup: $[\alpha]_{\mathrm{D}}-33.0^{\circ}\left(c \quad 1.0, \mathrm{CHCl}_{3}\right) ;{ }^{1} \mathrm{H}$ NMR (400 MHz, $\mathrm{CDCl}_{3}$, some characteristic signals are given): $\delta 6.17\left(\mathrm{dd}, 1 \mathrm{H}, J_{3,4}=J_{4,5}=9.9 \mathrm{~Hz}, \mathrm{H}-4\right)$, $6.13\left(\mathrm{dd}, 1 \mathrm{H}, J_{3,4}=J_{4,5}=9.8 \mathrm{~Hz}, \mathrm{H}-4\right), 6.08(\mathrm{dd}, 1 \mathrm{H}$, $\left.J_{3,4}=J_{4,5}=9.7 \mathrm{~Hz}, \mathrm{H}-4\right), 5.57\left(\mathrm{~d}, 1 \mathrm{H}, J_{1,2} 1.0 \mathrm{~Hz}\right.$, $\mathrm{H}-1), 5.48\left(\mathrm{~d}, 1 \mathrm{H}, J_{1,2} 1.5 \mathrm{~Hz}, \mathrm{H}-1\right), 5.36\left(\mathrm{~d}, 1 \mathrm{H}, J_{1,2} 1.1\right.$ $\mathrm{Hz}, \mathrm{H}-1), 5.32$ (d, $\left.1 \mathrm{H}, J_{1,2} 0.9 \mathrm{~Hz}, \mathrm{H}-1\right), 5.29(\mathrm{~d}, 1 \mathrm{H}$, $\left.J_{1,2} 0.7 \mathrm{~Hz}, \mathrm{H}-1\right), 5.24\left(\mathrm{~d}, 1 \mathrm{H}, J_{1,2} 0.6 \mathrm{~Hz}, \mathrm{H}-1\right), 5.18(\mathrm{~d}$, $\left.1 \mathrm{H}, \quad J_{1,2} 1.0 \mathrm{~Hz}, \mathrm{H}-1\right), 5.03\left(\mathrm{~d}, 1 \mathrm{H}, \quad J_{1,2} \quad 0.8\right.$ $\mathrm{Hz}, \mathrm{H}-1), 4.99$ (d, $\left.1 \mathrm{H}, J_{1,2} 1.2 \mathrm{~Hz}, \mathrm{H}-1\right), 2.16(\mathrm{~s}, 3 \mathrm{H}$, $\mathrm{CH}_{3} \mathrm{CO}$ ), 2.09 (s, $\left.3 \mathrm{H}, \mathrm{CH}_{3} \mathrm{CO}\right), 2.07$ (s, $3 \mathrm{H}, \mathrm{CH}_{3} \mathrm{CO}$ ), $1.99\left(\mathrm{~s}, 3 \mathrm{H}, \mathrm{CH}_{3} \mathrm{CO}\right), 1.81\left(\mathrm{~s}, 3 \mathrm{H}, \mathrm{CH}_{3} \mathrm{CO}\right) ;{ }^{13} \mathrm{C} \mathrm{NMR}$ 
$\left(100 \mathrm{MHz}, \mathrm{CDCl}_{3}\right): \delta 171.6,170.6,170.1,169.9,169.8$ (5 $\left.\mathrm{C}, 5 \mathrm{CH}_{3} \mathrm{CO}\right), \quad 166.9-164.9(\mathrm{PhCO}$, some signals overlapped), $118.0\left(\mathrm{CH}_{2}=\mathrm{CHCH}_{2} \mathrm{O}\right), 100.2,100.0,99.8$, 99.6, 99.3, 99.1, 98.6, 98.1, 97.7 (9 C, 9 C-1), 22.8, 21.1, 21.0, 20.8, 20.6 (5 C, C $\left.\mathrm{H}_{3} \mathrm{CO}\right)$. Anal. Calcd for $\mathrm{C}_{221} \mathrm{H}_{194} \mathrm{O}_{73}: \mathrm{C}, 66.06 ; \mathrm{H}, 4.86$. Found: $\mathrm{C}, 65.94 ; \mathrm{H}$, 5.01 .

3.19. Allyl 2,3,4,6-tetra- $O$-benzoyl- $\alpha$-D-mannopyranosyl$(1 \rightarrow 2)-3,4,6$-tri- $O$-benzoyl- $\alpha$-D-mannopyranosyl- $(1 \rightarrow 2)$ 3,4,6-tri- $O$-benzoyl- $\alpha$-D-mannopyranosyl-(1 $\rightarrow 6)-[2,3,4,6$ tetra- $O$-benzoyl- $\alpha$-D-mannopyranosyl- $(1 \rightarrow 3)-4,6$-di- $O$ acetyl-2- $O$-benzoyl- $\alpha$-D-mannopyranosyl- $(1 \rightarrow 2)-3,4,6-$ tri- $O$-benzoyl- $\alpha$-D-mannopyranosyl-( $1 \rightarrow 2)$-3,4-di- $O$ acetyl- $\alpha$-D-mannopyranosyl-(1 $\rightarrow 6)-[2,3,4,6$-tetra- $O$ benzoyl- $\alpha$-D-mannopyranosyl-( $1 \rightarrow 2)]-3$,4-di- $O$-acetyl- $\alpha$ D-mannopyranoside (22)

Compound 21 (161 mg, $0.04 \mathrm{mmol}$ ) was acetylated under the same conditions as that used for the preparation of $\mathbf{1 0}$ from $\mathbf{9}$, giving $\mathbf{2 2}(145 \mathrm{mg}, 89.5 \%)$ as a foamy solid: $[\alpha]_{\mathrm{D}}-36.5^{\circ}\left(c 1.0, \mathrm{CHCl}_{3}\right) ;{ }^{1} \mathrm{H}$ NMR $(400 \mathrm{MHz}$, $\mathrm{CDCl}_{3}$, some characteristic signals are given): $\delta 6.19$ $\left(\mathrm{dd}, 1 \mathrm{H}, J_{3,4}=J_{4,5}=9.8 \mathrm{~Hz}, \mathrm{H}-4\right), 6.12\left(\mathrm{dd}, 1 \mathrm{H}, J_{3,4}=\right.$ $\left.J_{4,5}=9.7 \mathrm{~Hz}, \mathrm{H}-4\right), 6.10\left(\mathrm{dd}, 1 \mathrm{H}, J_{3,4}=J_{4,5}=9.7 \mathrm{~Hz}\right.$, $\mathrm{H}-4), 6.07\left(\mathrm{dd}, 1 \mathrm{H}, J_{3,4}=J_{4,5}=9.7 \mathrm{~Hz}, \mathrm{H}-4\right), 5.78(\mathrm{dd}$, $\left.J_{1,2}=0.6 \mathrm{~Hz}, J_{2,3}=3.2 \mathrm{~Hz}, \mathrm{H}-2\right), 5.55\left(\mathrm{~d}, 1 \mathrm{H}, J_{1,2} 0.5\right.$ $\mathrm{Hz}, \mathrm{H}-1), 5.52\left(\mathrm{~d}, 1 \mathrm{H}, J_{1,2} 0.8 \mathrm{~Hz}, \mathrm{H}-1\right), 5.42(\mathrm{~d}, 1 \mathrm{H}$, $\left.J_{1,2} 0.8 \mathrm{~Hz}, \mathrm{H}-1\right), 5.37$ (d, $\left.1 \mathrm{H}, J_{1,2} 1.5 \mathrm{~Hz}, \mathrm{H}-1\right), 5.32$ $\left(\mathrm{dd}, 1 \mathrm{H}, J_{3,4}=J_{4,5}=9.9 \mathrm{~Hz}, \mathrm{H}-4\right), 5.31\left(\mathrm{~d}, 1 \mathrm{H}, J_{1,2} 0.8\right.$ $\mathrm{Hz}, \mathrm{H}-1), 5.16$ (d, $\left.1 \mathrm{H}, J_{1,2} 1.1 \mathrm{~Hz}, \mathrm{H}-1\right), 5.13(\mathrm{~d}, 1 \mathrm{H}$, $\left.J_{1,2} 0.9 \mathrm{~Hz}, \mathrm{H}-1\right), 5.05\left(\mathrm{~d}, 1 \mathrm{H}, J_{1,2} 0.9 \mathrm{~Hz}, \mathrm{H}-1\right), 4.95(\mathrm{~d}$, $\left.1 \mathrm{H}, J_{1,2} 1.2 \mathrm{~Hz}, \mathrm{H}-1\right), 2.17$ (s, $\left.3 \mathrm{H}, \mathrm{CH}_{3} \mathrm{CO}\right), 2.16$ (s, 3 $\left.\mathrm{H}, \mathrm{CH}_{3} \mathrm{CO}\right), 2.08\left(\mathrm{~s}, 3 \mathrm{H}, \mathrm{CH}_{3} \mathrm{CO}\right), 2.07(\mathrm{~s}, 3 \mathrm{H}$, $\left.\mathrm{CH}_{3} \mathrm{CO}\right), 2.03\left(\mathrm{~s}, 3 \mathrm{H}, \mathrm{CH}_{3} \mathrm{CO}\right), 1.99(\mathrm{~s}, 3 \mathrm{H}$, $\left.\mathrm{CH}_{3} \mathrm{CO}\right) ;{ }^{13} \mathrm{C}$ NMR $\left(100 \mathrm{MHz}, \mathrm{CDCl}_{3}\right): \delta$ 171.6, $170.5,170.3,170.2,169.9,169.6\left(6 \mathrm{C}, 6 \mathrm{CH}_{3} \mathrm{CO}\right)$, 167.2-164.9 ( $\mathrm{PhCO}$, some signals overlapped), 118.3 $\left(\mathrm{CH}_{2}=\mathrm{CHCH}_{2} \mathrm{O}\right), 100.3,100.1,99.8,99.7,99.3,99.0$, 98.8, 98.8, 97.7 (9 C, 9 C-1), 22.8, 20.9, 20.9, 20.8, 20.8, $20.4\left(6 \mathrm{C}, \mathrm{CH}_{3} \mathrm{CO}\right)$. Anal. Calcd for $\mathrm{C}_{223} \mathrm{H}_{196} \mathrm{O}_{74}$ : C, 65.97; H, 4.87. Found: C, 66.14; H, 5.10.

\subsection{Allyl $\alpha$-D-mannopyranosyl-( $(\rightarrow 2)-\alpha$-D-}

mannopyranosyl-(1 $\rightarrow 2)$ - $\alpha$-D-mannopyranosyl-(1 $\rightarrow 6)-[\alpha-$ D-mannopyranosyl-(1 $\rightarrow 3)$ - $\alpha$-D-mannopyranosyl-( $1 \rightarrow 2)$ $\alpha$-D-mannopyranosyl-(1 $\rightarrow 2)$ |- $\alpha$-D-mannopyranosyl- $(1 \rightarrow$ 6)-[ $\alpha$-D-mannopyranosyl-(1 $\rightarrow 2)]-\alpha$-D-mannopyranoside (23)

Compound 21 (700 mg, $0.17 \mathrm{mmol}$ ) was dissolved in a satd ammonia-MeOH solution $(50 \mathrm{~mL})$. After a week at $\mathrm{rt}$, the reaction mixture was concentrated, and the residue was purified by chromatography on Sephadex LH-20 (MeOH) to afford $23(181 \mathrm{mg}, 68.6 \%)$ as a syrup: $[\alpha]_{\mathrm{D}}+10.5^{\circ}\left(c 1.0, \mathrm{H}_{2} \mathrm{O}\right) ;{ }^{1} \mathrm{H}$ NMR $\left(400 \mathrm{MHz}, \mathrm{D}_{2} \mathrm{O}\right.$, some characteristic signals are given): $\delta 5.83(\mathrm{~m}, 1 \mathrm{H}$, $\left.\mathrm{OCH}_{2} \mathrm{CH}=\mathrm{CH}_{2}\right), 5.22\left(\mathrm{~m}, 1 \mathrm{H}, \mathrm{OCH}_{2} \mathrm{CH}=\mathrm{CH}_{2}\right), 5.16$ (s, $1 \mathrm{H}, \mathrm{H}-1), 5.16$ (s, $1 \mathrm{H}, \mathrm{H}-1), 5.14$ (s, $1 \mathrm{H}, \mathrm{H}-1), 5.01$ (d, $\left.1 \mathrm{H}, J_{1,2} 1.2 \mathrm{~Hz}, \mathrm{H}-1\right), 4.99$ (d, $1 \mathrm{H}, J_{1,2} 1.2 \mathrm{~Hz}, \mathrm{H}-1$ ), 4.98 (s, $1 \mathrm{H}, \mathrm{H}-1), 4.96$ (s, $1 \mathrm{H}, \mathrm{H}-1), 4.91$ (s, 1 H, H-1), $4.89\left(\mathrm{~m}, 1 \mathrm{H}, \mathrm{OCH}_{2} \mathrm{CH}=\mathrm{CH}_{2}\right), 4.88(\mathrm{~s}, 1 \mathrm{H}, \mathrm{H}-1) ;{ }^{13} \mathrm{C}$ NMR $\left(100 \mathrm{MHz}, \mathrm{D}_{2} \mathrm{O}\right): \delta 121.2\left(\mathrm{CH}_{2}=\mathrm{CHCH}_{2} \mathrm{O}\right)$, $104.9,104.8,104.8,104.7,103.2,103.2,100.7,100.6$, 100.2 (9 C, 9 C-1), 81.39, 81.38, 81.37, 81.02, 81.01, 80.51. MALDI-TOF MS Calcd for $\mathrm{C}_{57} \mathrm{H}_{96} \mathrm{O}_{46}: 1517.34$ [M]. Found: $1540.41[\mathrm{M}+\mathrm{Na}]$.

\section{Acknowledgements}

This work was supported by The Chinese Academy of Sciences (KZCX3-J-08) and by The National Natural Science Foundation of China (Projects 30070185 and 39970864).

\section{References}

1. Hodgson, R.; Peterson, W. H.; Riker, A. J. Phytopathology 1949, 39, 47-62.

2. Anagnostakis, S. L. Mycologia 1987, 79, 23-37.

3. Molinaro, A.; Piscopo, V.; Lanzetta, R.; Parrilli, M. Carbohydr. Res. 2002, 337, 1707-1714.

4. (a) Yi, Y.; Zhou, Z.; Ning, J.; Kong, F.; Li, J. Synthesis 2003, 4, 491-496;

(b) Wang, W.; Kong, F. Tetrahedron Lett. 1998, 19371940;

(c) Wang, W.; Kong, F. Carbohydr. Res. 1999, 315, 117127 ;

(d) Ning, J.; Yi, Y.; Kong, F. Tetrahedron Lett. 2002 , 5545-5549.

5. (a) Yang, G.; Kong, F. Synlett 2000, 1423-1426;

(b) Ning, J.; Zhang, W.; Yi, Y.; Yang, G.; Wu, Z.; Yi, J.; Kong, F. Bioorg. Med. Chem. 2003, in press.

6. (a) Ning, J.; Kong, F. Tetrahedron Lett. 1999, 1357-1360; (b) Heng, L.; Ning, J.; Kong, F. Carbohydr. Res. 2001, $331,431-438$;

(c) Ning, J.; Heng, L.; Kong, F. Tetrahedron Lett. 2002 , 673-675.

7. (a) Zhang, J.; Kong, F. Tetrahedron: Asymmetry 2002, 13, 243-252;

(b) Zhu, Y.; Kong, F. Synlett 2001, 1217-1220;

(c) Zhu, Y.; Kong, F. Synlett 2000, 1783-1787;

(d) Du, Y.; Zhang, M.; Kong, F. Tetrahedron 2001, 57 , 1757-1763;

(e) Zhu, Y.; Chen, L.; Kong, F. Chin. J. Chem. 2001, 19, 1289-1295.

8. (a) Zhu, Y.; Kong, F. Synlett 2000, 663-667;

(b) Zhu, Y.; Kong, F. Carbohydr. Res. 2001, 332, 1-21.

9. Ogawa, T.; Yamamoto, H. Carbohydr. Res. 1985, 137, 79-88.

10. Schmidt, R. R.; Kinzy, W. Adv. Carbohydr. Chem. Biochem. 1994, 50, 21-125. 